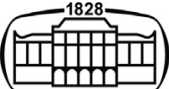

AKADÉMIAI KIADÓ

Acta Chromatographica

33 (2021) 3, 281-294

DOl:

$10.1556 / 1326.2020 .00777$

(c) 2020 The Authors

\section{Characterization and identification of flavonoids} from Bambusa chungii leaves extract by UPLC-ESI-Q-TOF-MS/MS

\author{
TING YUAN, XUE-FENG GUO*, SI-YUE SHAO, \\ RONG-MIAO AN, JIN WANG and JIA SUN
}

Key Laboratory of National Forestry and Grassland Administration, Beijing for Bamboo \& Rattan Science and Technology, Beijing 100102, PR China

Received: March 26, 2020 • Accepted: May 29, 2020

Published online: October 31, 2020

\begin{abstract}
Bamboo leaves extract (BLE) has a variety of physiological functions such as antitumour, anti-inflammatory, antioxidant and blood fat reduction activities and the flavonoids of bamboo leaves are the major active constituents. To profile the flavonoids in the complex BLE, a rapid and sensitive analytical method based on ultra-high-performance liquid chromatography coupled with electrospray ionization quadrupole time-of-flight tandem mass spectrometry (UPLC-ESI-Q-TOF-MS/MS) was developed for the structural identification of the flavonoids in Bambusa chungii leaves extract using accurate mass measurements and characteristic fragmentation patterns. After separation on an Agilent SB-C18 Rapid Resolution High Definition (RRHD) column $(2.1 \mathrm{~mm} \times 150 \mathrm{~mm}, 1.8 \mu \mathrm{m})$ by gradient elution with $0.1 \%$ formic acid aqueous solution and acetonitrile as the mobile phase, the sample was analysed by ESI-QTOF-MS/MS in the negative mode. A total of 22 flavonoids were detected, and eight of these were identified by comparison with reference standards, while the other fourteen were tentatively identified according to their MS/MS data. The main fragmentation pathways of flavonoid $C$-glycosides (compounds 1,5 and 10), flavonoid di-C,O-glycosides (compound 4), flavonoid di- $\mathrm{C}$-glycosides (compound 7) and flavonoid $\mathrm{C}, \mathrm{O}-$ di-glycosides (compounds 2 and 14) are shown in this article. This is the first report on the analysis of the flavonoids in the extract of $B$. chungii leaves. The present work demonstrates that UPLC-ESI-Q-TOF-MS/ MS is an efficient technique for identifying multiple flavonoids of BLE.
\end{abstract}

\section{KEYWORDS}

flavonoids, bamboo leaves extract (BLE), UPLC-ESI-Q-TOF-MS/MS, fragmentation pathway, Bambusa chungii

\section{INTRODUCTION}

Bamboo is a perennial evergreen plant, and it is a traditionally important commodity that can be used as a building material, process material, traditional medicine, food source, etc. [1]. In recent years, the research to advance the utilization of bamboo resources has been a very active field internationally. Bamboo has traditionally been an important ingredient in traditional Asian medicines, especially in China and India (Ayurveda). Bamboo leaves have a long history of consumption in China for their medicinal and nutritive properties, and they play a vital role in the food and pharmaceutical industries [2]. Bamboo leaves extract (BLE) has a variety of physiological functions and is a good source of natural antioxidants. BLE has been officially certified as a natural food additive by the Chinese Ministry of Health $[3,4]$. Flavonoids, phenolic acids, and coumaric lactones are the main bioactive components in BLE, and the flavonoid constituents are diverse and present at high contents [5-7].

Bambusa chungii is native to China, and the bamboo stalk surface is covered with a white powder; it is a relatively common clumping bamboo species in southern China and is one of several important clumping bamboo species [8]. However, there has been little research on the BLE of B. chungii. The flavonoids of bamboo leaves are the main active constituents of 
BLE, and they have free radicals scavenging, antioxidation, anti-inflammatory, lipid-lowering, anti-depressant and wound healing activities [9-14]. At present, the flavonoids of bamboo leaves have been marketed as health care products, suggesting their great development potential and research value.

UPLC-ESI-Q-TOF-MS/MS has been recognized as a powerful tool for the characterization of complex extracts, resulting in its widespread used [15-17]. ESI is a soft ionization method that provides protonated and deprotonated molecules. Q-TOF-MS combines high sensitivity and mass accuracy for both precursor and product ions, and thus, the elemental composition of the parent and fragment ions can be quickly and efficiently confirmed [18]. These features can greatly facilitate the prediction of elemental compositions and fragmentation pathways [19]. Recently, chromatographic separation coupled with mass spectrometry (MS) has been identified as a powerful technique for identifying compounds, including identification and structural studies of flavonoid glycosides [20]. The fragmentation pathways of flavonoid $O$-glycosides mainly involve losing glycoside moieties at the glycosidic $O$-linkages through a dehydration condensation reaction that forms high-abundance flavonoid cores and retro Diels-Adler (RDA) reactions [21]. The fragmentation patterns of flavonoid $\mathrm{C}$-glycosides mainly involve the loss of $\mathrm{H}_{2} \mathrm{O}$, the cross-ring cleavage of the aglycone moiety and RDA reactions [22]. An efficient and sensitive analytical method using ultra-high-performance liquid chromatography/quadrupole time-of-flight mass spectrometry (UPLC-ESI-Q-TOF-MS/MS) was used to identify the flavonoids in $B$. chungii leaves extract. This technique offers high efficiency, high sensitivity, short analysis times and high precision in the $\mathrm{m} / z$ value, and it has been successfully applied in the identification of flavonoids in other plant extracts [23-26].

In this study, an analytical method for the identification or tentative identification of 22 flavonoids in the leaves extract of $B$. chungii was successfully established using UPLC-ESI-Q-TOF-MS/MS, allowing us to explore mass spectrometric fragmentation pathways based on accurate mass measurements and the data found in the literature. Our results provide interesting and valuable MS/MS data and reliable information for the future rapid identification of similar types of flavonoids in plants or other medicinal extracts.

\section{EXPERIMENTAL}

\section{Chemicals and materials}

LC/MS-grade methanol, acetonitrile and formic acid were purchased from the Sigma Chemical Co (Sigma-Aldrich, St. Louis, MO, USA). AR-grade ethanol and petroleum ether were used in the preparation of the extract (Merck, Darmstadt, Germany). Ultrapure water was obtained from the Quchenshi Group Co., Ltd. (Xianggang, China).
Bamboo (B. chungii) leaves were collected from Nanchang, Jiangxi Province, China. B. chungii leaves were confirmed the authenticity by Fuming Xiao of Jiangxi Academy of Forestry, a researcher with a Ph.D. in forest cultivation. The fresh leaves were dried in the shade at room temperature $\left(20-25{ }^{\circ} \mathrm{C}\right)$ and then powdered. A voucher specimen was deposited at the International Centre for Bamboo and Rattan (ICBR), Beijing, China.

Eight reference standards, namely, luteolin-6- $C$-glucoside (1), luteolin-6-C-glucosyl-2" -O-rhamnoside (2), apigenin-6$C$-arabinosyl-7-O-glucoside (4), apigenin-6-C-glucoside (5), apigenin-6- $C$-arabinosyl-8- $C$-arabinoside (7), luteolin-7-Oglucoside (8), luteolin-6- $C$-arabinoside (10) and apigenin-6$\mathrm{C}$-arabinosyl-2"-O-rhamnoside (14), were isolated in our laboratory.

\section{Extraction, isolation, and sample preparation}

The leaves of bamboo ( $5 \mathrm{~kg}$ ) were shade-dried and then extracted three times with $95 \%$ ethanol by cold percolation. The residue was obtained after evaporation of the solvent. The residue was suspended in $\mathrm{H}_{2} \mathrm{O}$ and partitioned between water and petroleum ether. The water was removed from the aqueous fraction, and the residue ( $277 \mathrm{~g}$ ) was subjected to macroporous resin Diaion HP-20 (Mitsubishi Chemical Corp, Tokyo, Japan), Rp-18 (50 $\mu \mathrm{m}, \mathrm{YMC}$ ), and Sephadex LH-20 (Pharmacia Fine Chemicals, Uppsala, Sweden) columns eluted with $40 \%$ ethanol. After evaporation of the solvent, a solid residue of $181 \mathrm{~g} 40 \%$ ethanol fraction was obtained. Then, the $40 \%$ ethanol fraction was applied to a preparative High Performance Liquid Chromatography (HPLC) using a Shimadzu LC-6 AD instrument with an SPD-20A detector (Shimadzu, Kyoto, Japan) and a YMC-Pack ODS-A column $(250 \times 20 \mathrm{~mm}, 5 \mu \mathrm{m}$, YMC, Kyoto, Japan $)$ and eluted with acetonitrile-water $(14: 86, \mathrm{v} / \mathrm{v})$ to yield compounds $1,2,4,5,7$, 8,10 and 14. All of these compounds were identified by NMR and MS techniques using Bruker 300 NMR spectrometer (Bruker, Zurich, Switzerland) and Agilent 6540 high-resolution time-of-flight (Q-TOF) mass spectrometer (Agilent Technologies, Singapore) respectively.

The purities of these compounds were determined to be higher than $95 \%$ by HPLC-Photo-Diode Array (PDA) analysis using a Waters 2695 system and a 2996 PDA detector (Waters, Milford, MA, USA) with a YMC-Pack ODS-AQ C18 column $(250 \times 4.6 \mathrm{~mm}, 5 \mu \mathrm{m}$, YMC). All the reference standards were dissolved in methanol to prepare solutions with final concentrations of $0.02 \mathrm{mg} / \mathrm{mL}$ for UPLC-QTOFMS/MS analysis. A solid sample of $0.05 \mathrm{~g}$ of the dried $40 \%$ ethanol fraction was weighted, dissolved in methanol-water $(1: 9, \mathrm{v} / \mathrm{v})$ to prepare a $5 \mathrm{mg} / \mathrm{mL}$ stock solution. Then the solution was filtered through a $0.2 \mu \mathrm{m}$ polytetrafluoroethylene (PTFE) syringe filter, and the filtrate $(10 \mu \mathrm{L})$ was subjected to UPLC-ESI-Q-TOF-MS/MS analysis.

\section{NMR analysis}

The ${ }^{1} \mathrm{H}$ and ${ }^{13} \mathrm{C}$ nuclear magnetic resonance (NMR) spectra were determined with a Bruker 300 NMR spectrometer (Bruker, Zurich, Switzerland) at $300 \mathrm{MHz}$ using Dimethyl 
sulfoxide- $d_{6}$ (DMSO- $\left.d_{6}\right)$ as the solvent and tetramethylsilane (TMS) as an internal standard. Chemical shifts were expressed in $\delta(\mathrm{ppm})$, and coupling constants were reported in hertz. The ${ }^{1} \mathrm{H}$ and ${ }^{13} \mathrm{C}$ NMR spectra of eight reference standards were included in Supporting information.

\section{Instruments and analytical conditions}

Analyses were performed with an Agilent UPLC 1290 system (Agilent Technologies, Singapore) coupled with a highresolution quadrupole time-of-flight (QTOF) MS/MS 6540 system (Agilent Technologies, Singapore) equipped with a JetStream technology ESI ion source that was operated in the negative ionization mode.

For chromatographic analysis, a $2.1 \mathrm{~mm} \times 150 \mathrm{~mm}$ Agilent SB-C18 Rapid Resolution High Definition (RRHD) column with a particle size of $1.8 \mu \mathrm{m}$ was used. For analyses conducted in negative ion mode, mobile phase A consisted of $0.1 \%(\mathrm{v} / \mathrm{v})$ formic acid in water, and mobile phase B consisted of acetonitrile. The linear gradient elution program was optimized follows: $90-85 \%$ mobile phase A (0-30 $\mathrm{min}), 85-85 \%$ mobile phase A (30-40 min), $85-80 \%$ mobile phase A (40-60 $\mathrm{min})$ and $80-70 \%$ mobile phase $\mathrm{A}(60-80 \mathrm{~min})$. The flow rate was set at $250 \mu \mathrm{L} / \mathrm{min}$ and the column temperature was maintained at $40{ }^{\circ} \mathrm{C}$. The wavelength for UV detection and the injection volume were set at $350 \mathrm{~nm}$ and $1 \mu \mathrm{L}$, respectively.

The mass spectrometer was equipped with a JetStream technology ESI source. The source parameters were as follows for the negative ionization mode: gas temperature, 350 ${ }^{\circ} \mathrm{C}$; drying gas flow rate, $8 \mathrm{~L} / \mathrm{min}$; nebulizer pressure, $35 \mathrm{psi}$; sheath gas temperature, $350{ }^{\circ} \mathrm{C}$; sheath gas flow rate, $11 \mathrm{~L} /$ min; VCap, $3500 \mathrm{~V}$; nozzle voltage (Expt), $1000 \mathrm{~V}$; fragmentor voltage, $175 \mathrm{~V}$; skimmer voltage, $65 \mathrm{~V}$; OCT $1 \mathrm{RF}$ $\mathrm{Vpp}, 750 \mathrm{~V}$; collision energy, 15-45 eV (in step of $10 \mathrm{eV}$ ); collision gas, $\mathrm{N}_{2}$; and collision cell, hexapole. The scanning range was $m / z 50-1,100$ for the MS scan and $m / z 50-800$ for the MS/MS scan.

\section{RESULTS AND DISCUSSION}

\section{UPLC-ESI-Q-TOF-MS/MS analysis of the Bambusa chungii leaves extract}

UPLC-UV chromatograms collected at $350 \mathrm{~nm}$ and UPLCESI-Q-TOF-MS/MS total ion chromatograms (TICs) of the major flavonoids in the $B$. chungii leaves extract are shown in Fig. 1. A total of 22 flavonoids were identified, including 4 flavonoid C-glycosides, 2 flavonoid $O$-glycosides, 1 flavonoid di- $C$-glycoside, 3 flavonoid di- $C, O$-glycosides and 12 flavonoid $\mathrm{O}, \mathrm{C}$-di-glucosides. Their structures are shown in Fig. 2. Excellent mass accuracy and resolution were achieved in both the MS and MS/MS scans. High mass accuracy in MS/ MS greatly facilitates structural elucidation, as it allows unambiguous assignment of the elemental composition. The major flavonoids characterized in the $B$. chungii leaves extract by UPLC-ESI-Q-TOF-MS/MS are shown in Table 1, and the theoretical and experimental masses, relative errors and relative abundances, retention times (RTs), compound names, formulae of the deprotonated molecules and fragment ions of these major flavonoids are summarized. For structural identification and determining the mass spectrometry fragmentation pathways of various types of flavonoids and their glycosides in $B$. chungii leaves extract, known compounds were analysed by quadrupole time-offlight tandem mass spectrometry (QTOF-MS/MS) in the negative ion mode to determine the specificity of the MS/MS spectral pattern of each structure. Then, the structures of the unknown compounds were tentatively determined by interpretation of their RTs, MS and MS/MS spectra and comparison with the data provided in the literature. Analysis of the ions obtained by MS fragmentation as well as their relative abundances can provide very valuable information to facilitate the tentative identification of their chemical structures. The nomenclature of product ions created after

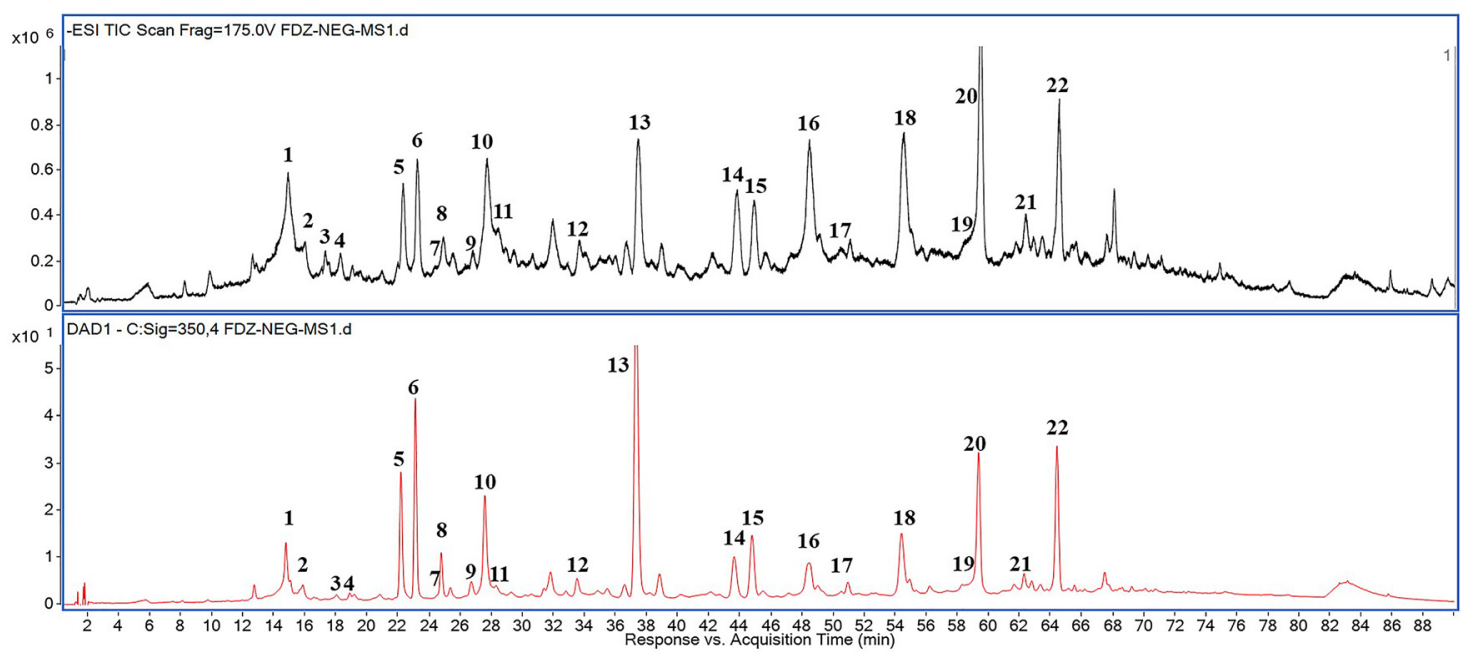

Fig. 1. UPLC-UV chromatogram acquired at $350 \mathrm{~nm}$ and UPLC-ESI-Q-TOF-MS/MS total ion chromatogram (TIC) of the major constituents in the Bambusa chungii leaves extract 


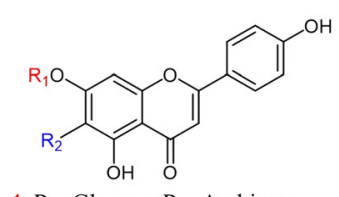

4: $\mathrm{R}_{1}=$ Glucose, $\mathrm{R}_{2}=$ Arabinose

5: $\mathrm{R}_{1}=\mathrm{H}, \mathrm{R}_{2}=$ Glucose

7: $\mathrm{R}_{1}=$ Arabinose, $\mathrm{R}_{2}=$ Arabinose<smiles>[R6]Oc1c([2H])c(O)c(C(=O)O)c(Br)c1[2H]</smiles><smiles>CC=Cc1cccc(O)c1</smiles>

1: $\mathrm{R}_{1}=\mathrm{H}, \mathrm{R}_{2}=\mathrm{H}, \mathrm{R}_{3}=$ Glucose, $\mathrm{R}_{4}=\mathrm{H}, \mathrm{R}_{5}=\mathrm{H}$ 3: $\mathrm{R}_{1}=$ Rhamnose, $\mathrm{R}_{2}=\mathrm{H}, \mathrm{R}_{3}=\mathrm{H}, \mathrm{R}_{4}=\mathrm{H}, \mathrm{R}_{5}=$ Glucos 8: $\mathrm{R}_{1}=\mathrm{H}, \mathrm{R}_{2}=$ Glucose, $\mathrm{R}_{3}=\mathrm{H}, \mathrm{R}_{4}=\mathrm{H}, \mathrm{R}_{5}=\mathrm{H}$ 9: $\mathrm{R}_{1}=\mathrm{H}, \mathrm{R}_{2}=\mathrm{H}, \mathrm{R}_{3}=$ Arabinose, $\mathrm{R}_{4}=$ Glucose, $\mathrm{R}_{5}=\mathrm{H}$ 10: $\mathrm{R}_{1}=\mathrm{H}, \mathrm{R}_{2}=\mathrm{H}, \mathrm{R}_{3}=$ Arabinose, $\mathrm{R}_{4}=\mathrm{H}, \mathrm{R}_{5}=\mathrm{H}$ 17: $\mathrm{R}_{1}=$ Rhamnose, $\mathrm{R}_{2}=\mathrm{H}, \mathrm{R}_{3}=\mathrm{H}, \mathrm{R}_{4}=\mathrm{H}, \mathrm{R}_{5}=\mathrm{H}$<smiles>CC1OC(c2c(O)cc3oc(-c4ccc(O)c(O)c4)cc(=O)c3c2O)CC(OC2OC(CO)C(O)C(O)C2O)C1O</smiles><smiles>CC1OC(O/C=C/c2c(O)cc3oc(-c4ccc(O)c(O)c4)cc(=O)c3c2O)C(O)C(O)C1O</smiles><smiles>CC1OC(c2c(O)cc3oc(-c4ccc(O)cc4)cc(=O)c3c2O)C(OC2OC(C)C(O)C(O)C2O)C(=O)C1O</smiles><smiles>CC1OC(c2c(O)cc(O)c3c(=O)cc(-c4ccc(O)c(O)c4)oc23)C(OC2OC(C)C(O)C(O)C2O)C(=O)C1O</smiles>

15

2: $\mathrm{R}_{1}=\mathrm{CH}_{2} \mathrm{OH}, \mathrm{R}_{2}=$ Rhamnose, $\mathrm{R}_{3}=\mathrm{OH}$ 6: $\mathrm{R}_{1}=\mathrm{CH}_{2} \mathrm{OH}, \mathrm{R}_{2}=$ Rhamnose, $\mathrm{R}_{3}=\mathrm{H}$ 11: $\mathrm{R}_{1}=\mathrm{H}, \mathrm{R}_{2}=$ Rhamnose, $\mathrm{R}_{3}=\mathrm{OH}$ 14: $\mathrm{R}_{1}=\mathrm{H}, \mathrm{R}_{2}=$ Rhamnose, $\mathrm{R}_{3}=\mathrm{H}$ 18: $\mathrm{R}_{1}=\mathrm{CH}_{3}, \mathrm{R}_{2}=$ Rhamnose, $\mathrm{R}_{3}=\mathrm{O}$<smiles>CC1OC(c2c(O)cc3oc(-c4ccc(O)c(O)c4)cc(=O)c3c2O)C(OC2OC(C)C(O)C(O)C2O)C(=O)C1O</smiles>

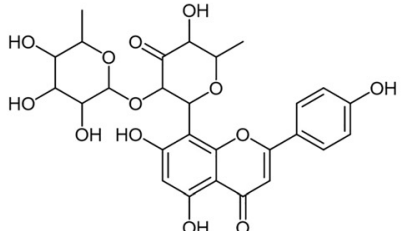

20

21

Fig. 2. Structures of flavonoids $1-22$

the cleavage of the glycosidic bonds follows that proposed by Domon and Costello [27] and Ma et al. [28] (Scheme 1).

\section{Q-TOF-MS/MS ANALYSIS OF THE MAJOR FLAVONOIDS IN THE BAMBUSA CHUNGII LEAVES EXTRACT}

\section{Identification of C-glycosides}

Compounds $1(\mathrm{RT}=14.86 \mathrm{~min}), 5(\mathrm{RT}=22.21 \mathrm{~min})$ and 10 $(\mathrm{RT}=28.28 \mathrm{~min})$ were positively identified as luteolin-6- $C$ glucoside, apigenin-6- $C$-glucoside and luteolin-6- $C$-arabinoside, respectively, by comparison of their RTs and mass spectral analysis with the reference standards. Compound 1 $\left(\mathrm{C}_{21} \mathrm{H}_{20} \mathrm{O}_{11}\right)$ yielded a deprotonated ion $[\mathrm{M}-\mathrm{H}]^{-}$at $\mathrm{m} / z$ 447.0916. The tandem mass spectrum showed a fragment ion at $\mathrm{m} / z 429.0805[\mathrm{M}-\mathrm{H}-18]^{-}$, which corresponded to the loss of a molecule of $\mathrm{H}_{2} \mathrm{O}$ between the $2^{\prime \prime}$-hydroxyl group of the sugar and the 5 or 7-hydroxyl group of the aglycone. The fragment ions at $m / z 357.0609\left({ }^{0,3} \mathrm{X}_{0}\right)[\mathrm{M}-$ $\mathrm{H}-90]^{-}, m / z 327.0510\left({ }^{0,2} \mathrm{X}_{0}\right) \quad[\mathrm{M}-\mathrm{H}-120]^{-}$and $\mathrm{m} / z$ $297.0398\left({ }^{0,1} \mathrm{X}_{0}\right)[\mathrm{M}-\mathrm{H}-90]^{-}$indicate the losses of $90 \mathrm{Da}$, $120 \mathrm{Da}$ and $150 \mathrm{Da}$ from the $[\mathrm{M}-\mathrm{H}]^{-}$ion, respectively, which were characteristic of the cross-ring cleavage of the glucose part of the flavonoid glycosyl moiety. In addition, the fragment ion at $\mathrm{m} / z 285.0399\left[\mathrm{Y}_{0}\right]^{-}$indicated that compound 1 originated from luteolin and produced a fragment ion at $m / z 133.0270\left[{ }^{1,3} \mathrm{~B}_{0}\right]^{-}$by retro-Diels-Alder
(RDA) cleavage. Compound $5\left(\mathrm{C}_{21} \mathrm{H}_{20} \mathrm{O}_{10}\right)$ was observed at $\mathrm{m} / z 431.0971\left([\mathrm{M}-\mathrm{H}]^{-}\right.$ion) in ESI-MS. Similarly, it produced a series of fragment ions at $m / z 413.0886$ [M-H$18]^{-}, 341.0663\left({ }^{0,3} \mathrm{X}_{0}\right), 311.0561\left({ }^{0,2} \mathrm{X}_{0}\right)$ and $281.0455\left({ }^{0,1} \mathrm{X}_{0}\right)$ by the diagnostic neutral losses of $18,90,120$ and $150 \mathrm{Da}$ from the deprotonated ion, respectively, which were $16 \mathrm{Da}$ lighter than those of compound 1. The most abundant ions were at $\mathrm{m} / z 341.0663$ and $\mathrm{m} / z 311.0561$ (as shown in Table 1), and their relative intensities were 47 and 100\%, respectively, at CE $25 \mathrm{eV}$. In addition, compound 5 gave a deprotonated aglycone fragment at $\mathrm{m} / z 269.0445\left[\mathrm{Y}_{0}\right]^{-}$, indicating that the flavonoid core was apigenin and its fragment ions at $m / z 117.0323\left[{ }^{1,3} \mathrm{~B}_{0}\right]^{-}$was produced by an RDA reaction. Compound $10 \quad\left(\mathrm{C}_{20} \mathrm{H}_{18} \mathrm{O}_{10}\right)$ yielded a deprotonated ion $[\mathrm{M}-\mathrm{H}]^{-}$at $\mathrm{m} / z$ 417.0838. The ion at $\mathrm{m} / z$ $399.0727[\mathrm{M}-\mathrm{H}-18]^{-}$indicated the loss of water from the deprotonated ion. In the $\mathrm{MS}^{2}$ spectrum of compound 10 , the fragment ions at $m / z 357.0650\left({ }^{0,3} \mathrm{X}_{0}\right), m / z 327.0522\left({ }^{0,2} \mathrm{X}_{0}\right)$ and $m / z 297.0431\left({ }^{0,1} \mathrm{X}_{0}\right)$ were due to the losses of 60,90 and $120 \mathrm{Da}$, respectively, from the $[\mathrm{M}-\mathrm{H}]^{-}$ion through crossring cleavages of the arabinosyl unit. The deprotonated aglycone fragment at $\mathrm{m} / z 285.0384\left[\mathrm{Y}_{0}\right]^{-}$, indicated that the flavonoid core was luteolin. The fragmentation schemes of compounds 1, 5 and 10 are shown in Scheme 2.

The fragmentation patterns of flavonoid $C$-glycosides mainly involve the losses of $\mathrm{H}_{2} \mathrm{O}$ between the $2^{\prime \prime}$-hydroxyl group of the sugar and the 5- or 7-hydroxyl group of the aglycone and the cross-ring cleavage of the aglycone moiety. The fragments of the studied $[\mathrm{M}-\mathrm{H}]^{-}$ions of $C$-glycosides 
Table 1. The major flavonoids characterized in Bambusa chungii leaves extract by UPLC/Q-TOF-MS

\begin{tabular}{|c|c|c|c|c|c|c|c|c|}
\hline No. & $\begin{array}{l}\text { Retention } \\
\text { time (min) }\end{array}$ & $\begin{array}{l}\text { Molecular } \\
\text { formula }\end{array}$ & $\begin{array}{l}\text { Theoretical } \\
{[\mathrm{M}-\mathrm{H}]^{-} \mathrm{m} / z}\end{array}$ & $\begin{array}{l}\text { Experimental } \\
{[\mathrm{M}-\mathrm{H}]^{-} \mathrm{m} / z}\end{array}$ & $\begin{array}{l}\text { Error } \\
(\mathrm{ppm})\end{array}$ & $\begin{array}{l}\mathrm{CE} \\
(\mathrm{eV})\end{array}$ & Fragment ions & $\begin{array}{c}\text { Tentative } \\
\text { identification }\end{array}$ \\
\hline 1 & 14.86 & $\mathrm{C}_{21} \mathrm{H}_{20} \mathrm{O}_{11}$ & 447.0933 & $447.0916(6.71)$ & 3.76 & 25 & $\begin{array}{c}429.0805(7), \\
387.0706(2), \\
357.0609(100), \\
327.0510(80), \\
297.0398(21), \\
285.0399(10), \\
133.0270(1)\end{array}$ & $\begin{array}{l}\text { Luteolin-6-C- } \\
\text { glucoside } \\
\text { (isoorientin) }^{\mathrm{a}}\end{array}$ \\
\hline 2 & 15.87 & $\mathrm{C}_{27} \mathrm{H}_{30} \mathrm{O}_{15}$ & 593.1512 & $\begin{array}{c}593.1514 \\
(14.09)\end{array}$ & -0.35 & 35 & $\begin{array}{c}473.1106(100), \\
447.0898(1), \\
429.0841(16), \\
369.0614(5), \\
357.0632(26), \\
339.0516(12), \\
327.0514(29), \\
309.0422(20), \\
285.0403(7)\end{array}$ & $\begin{array}{l}\text { Luteolin-6-C- } \\
\text { glucosyl-2" }-O- \\
\text { rhamnoside }^{\text {a }}\end{array}$ \\
\hline 3 & 18.47 & $\mathrm{C}_{27} \mathrm{H}_{30} \mathrm{O}_{15}$ & 593.1512 & $\begin{array}{c}593.1501 \\
(90.83)\end{array}$ & 1.84 & 35 & $\begin{array}{l}431.1003(100), \\
357.0636(43), \\
327.0550(58), \\
311.0596(59), \\
285.0442(27)\end{array}$ & $\begin{array}{l}\text { Luteolin-8-C- } \\
\text { rhamnosyl- } 4^{\prime}-O- \\
\text { glucoside }\end{array}$ \\
\hline 4 & 18.53 & $\mathrm{C}_{26} \mathrm{H}_{28} \mathrm{O}_{14}$ & 563.1406 & $563.1390(100)$ & 2.89 & 35 & $\begin{array}{l}473.1109(47), \\
401.0837(69), \\
341.0642(31), \\
311.0546(97), \\
297.0407(83)\end{array}$ & $\begin{array}{l}\text { Apigenin-6-C- } \\
\text { arabinosyl-7-O- } \\
\text { glucoside }^{\mathrm{a}}\end{array}$ \\
\hline 5 & 22.21 & $\mathrm{C}_{21} \mathrm{H}_{20} \mathrm{O}_{10}$ & 431.0984 & $431.0971(6.27)$ & 2.94 & 25 & $\begin{array}{c}413.0886(2), \\
371.0751(1), \\
341.0663(47), \\
323.0550(5), \\
311.0561(100), \\
281.0455(4), \\
269.0445(6), \\
117.0323(1)\end{array}$ & $\begin{array}{c}\text { Apigenin-6-C- } \\
\text { glucoside (isovitexin) }\end{array}$ \\
\hline 6 & 23.20 & $\mathrm{C}_{27} \mathrm{H}_{30} \mathrm{O}_{14}$ & 577.1563 & $577.1553(8.76)$ & 1.69 & 35 & $\begin{array}{c}457.1134(12), \\
431.1001(1), \\
413.0873(35), \\
353.0671(9), \\
341.0658(16), \\
323.0552(10), \\
311.0561(15), \\
293.0452(100), \\
269.0401(3), \\
117.0309(1)\end{array}$ & $\begin{array}{l}\text { Apigenin-6-C- } \\
\text { glucosyl-2" }-O- \\
\text { rhamnoside }\end{array}$ \\
\hline 7 & 24.57 & $\mathrm{C}_{25} \mathrm{H}_{26} \mathrm{O}_{13}$ & 533.1301 & $\begin{array}{c}533.1285 \\
(30.91)\end{array}$ & 2.93 & 35 & $\begin{array}{c}473.1077(42), \\
443.0977(47), \\
413.0856(16), \\
383.0776(100), \\
353.0657(75), \\
269.0418(3)\end{array}$ & $\begin{array}{l}\text { Apigenin-6,8-C- } \\
\text { arabinoside }^{\mathrm{a}}\end{array}$ \\
\hline 8 & 25.16 & $\mathrm{C}_{21} \mathrm{H}_{20} \mathrm{O}_{11}$ & 447.0933 & 447.0953 (3.38) & -4.5 & 35 & $\begin{array}{c}285.0423(100), \\
284.0338(68), \\
151.0064(1), \\
133.0291(2)\end{array}$ & $\begin{array}{l}\text { Luteolin-7-O- } \\
\text { glucoside }^{\mathrm{a}}\end{array}$ \\
\hline 9 & 27.22 & $\mathrm{C}_{27} \mathrm{H}_{30} \mathrm{O}_{14}$ & 577.1563 & $577.1592(1.65)$ & -5.05 & 35 & $\begin{array}{c}473.1144(4) \\
415.1053(18) \\
397.0957(7) \\
371.0789(8)\end{array}$ & $\begin{array}{l}\text { Luteolin-6-C- } \\
\text { boivinosyl-7-O- } \\
\text { glucoside }\end{array}$ \\
\hline
\end{tabular}


Table 1. Continued

\begin{tabular}{|c|c|c|c|c|c|c|c|c|}
\hline No. & $\begin{array}{l}\text { Retention } \\
\text { time (min) }\end{array}$ & $\begin{array}{l}\text { Molecular } \\
\text { formula }\end{array}$ & $\begin{array}{l}\text { Theoretical } \\
{[\mathrm{M}-\mathrm{H}]^{-} \quad m / z}\end{array}$ & $\begin{array}{l}\text { Experimental } \\
{[\mathrm{M}-\mathrm{H}]^{-} \quad m / z}\end{array}$ & $\begin{array}{l}\text { Error } \\
(\mathrm{ppm})\end{array}$ & $\begin{array}{l}\mathrm{CE} \\
(\mathrm{eV})\end{array}$ & Fragment ions & $\begin{array}{c}\text { Tentative } \\
\text { identification }\end{array}$ \\
\hline & & & & & & & $\begin{array}{c}353.0690(52), \\
341.0674(12), \\
311.0577(100), \\
285.0419(8), \\
133.0304(1)\end{array}$ & \\
\hline 10 & 28.28 & $\mathrm{C}_{20} \mathrm{H}_{18} \mathrm{O}_{10}$ & 417.0827 & $\begin{array}{c}417.0838 \\
(12.19)\end{array}$ & -2.58 & 25 & $\begin{array}{c}399.0727(8), \\
357.0650(100), \\
327.0522(38), \\
297.0431(21), \\
285.0384(14)\end{array}$ & $\begin{array}{l}\text { Luteolin-6-C- } \\
\text { arabinoside }^{\mathrm{a}}\end{array}$ \\
\hline 11 & 31.92 & $\mathrm{C}_{26} \mathrm{H}_{28} \mathrm{O}_{14}$ & 563.1406 & $\begin{array}{c}563.1416 \\
(31.41)\end{array}$ & -1.72 & 35 & $\begin{array}{c}473.1065(100), \\
417.0800(11), \\
399.0706(32), \\
357.0598(25), \\
339.0493(26), \\
327.0485(41), \\
309.0388(26), \\
298.0478(77), \\
285.0398(13)\end{array}$ & $\begin{array}{l}\text { Luteolin-6-C- } \\
\text { arabinosyl-2"-O- } \\
\text { rhamnoside }\end{array}$ \\
\hline 12 & 37.44 & $\mathrm{C}_{27} \mathrm{H}_{30} \mathrm{O}_{13}$ & 561.1614 & $561.1615(6.81)$ & -0.24 & 45 & $\begin{array}{c}457.1171(17), \\
399.1108(5), \\
381.0928(11), \\
355.0850(3), \\
337.0744(31), \\
295.0630(100), \\
281.0473(9), \\
269.0482(12), \\
117.0340(2)\end{array}$ & $\begin{array}{l}\text { Apigenin-6-C- } \\
\text { boivonosyl-3"- }-O \text { - } \\
\text { glucoside }\end{array}$ \\
\hline 13 & 39.11 & $\mathrm{C}_{27} \mathrm{H}_{30} \mathrm{O}_{14}$ & 577.1563 & $\begin{array}{c}577.1587 \\
(25.67)\end{array}$ & -4.19 & 35 & $\begin{array}{c}473.1082(83), \\
431.0949(8), \\
413.0924(23), \\
357.0638(48), \\
339.0505(21), \\
327.0521(48), \\
309.0383(21), \\
298.0511(100), \\
285.0432(19)\end{array}$ & $\begin{array}{l}\text { Luteolin-8-C- } \\
\text { rhamnosyl-2"-O- } \\
\text { rhamnoside }\end{array}$ \\
\hline 14 & 44.77 & $\mathrm{C}_{26} \mathrm{H}_{28} \mathrm{O}_{13}$ & 547.1457 & $\begin{array}{c}547.1439 \\
(14.06)\end{array}$ & 3.39 & 35 & $\begin{array}{c}457.1140(12), \\
401.0877(4), \\
383.0763(83), \\
341.0659(31), \\
323.0548(29), \\
311.0553(30), \\
293.0443(87), \\
269.0446(12)\end{array}$ & $\begin{array}{l}\text { Apigenin-6-C- } \\
\text { arabinosyl- } 2^{\prime \prime}-O- \\
\text { rhamnoside }^{\mathrm{a}}\end{array}$ \\
\hline 15 & 45.54 & $\mathrm{C}_{27} \mathrm{H}_{28} \mathrm{O}_{14}$ & 575.1406 & $575.1397(9.27)$ & 1.61 & 35 & $\begin{array}{c}473.1080(7), \\
429.0853(12), \\
411.0721(28), \\
385.0567(15), \\
367.0467(40) \\
325.0362(100), \\
298.0485(53), \\
285.0408(18) \\
133.0297(2)\end{array}$ & $\begin{array}{c}\text { Cassiaoccidentalin B } \\
\text { isomer }\end{array}$ \\
\hline 16 & 48.44 & $\mathrm{C}_{27} \mathrm{H}_{28} \mathrm{O}_{14}$ & 575.1406 & $575.1383(7.22)$ & 4.04 & 35 & $\begin{array}{l}473.1071(10) \\
429.0812(13), \\
411.0709(35) \\
385.0546(19)\end{array}$ & Cassiaoccidentalin B \\
\hline
\end{tabular}

(continued) 
Table 1. Continued

\begin{tabular}{|c|c|c|c|c|c|c|c|c|}
\hline No. & $\begin{array}{l}\text { Retention } \\
\text { time (min) }\end{array}$ & $\begin{array}{l}\text { Molecular } \\
\text { formula }\end{array}$ & $\begin{array}{l}\text { Theoretical } \\
{[\mathrm{M}-\mathrm{H}]^{-} \mathrm{m} / z}\end{array}$ & $\begin{array}{l}\text { Experimental } \\
{[\mathrm{M}-\mathrm{H}]^{-} m / z}\end{array}$ & $\begin{array}{l}\text { Error } \\
(\mathrm{ppm})\end{array}$ & $\begin{array}{l}\mathrm{CE} \\
(\mathrm{eV})\end{array}$ & Fragment ions & $\begin{array}{c}\text { Tentative } \\
\text { identification }\end{array}$ \\
\hline & & & & & & & $\begin{array}{c}367.0455(59), \\
325.0347(100), \\
298.0478(46), \\
285.0395(29)\end{array}$ & \\
\hline 17 & 50.61 & $\mathrm{C}_{21} \mathrm{H}_{20} \mathrm{O}_{10}$ & 431.0984 & $\begin{array}{c}431.0988 \\
(14.85)\end{array}$ & -0.99 & 25 & $\begin{array}{c}413.0883(11) \\
357.0619(100), \\
327.0515(59), \\
297.0393(17) \\
285.0398(10)\end{array}$ & $\begin{array}{l}\text { Luteolin-8-C- } \\
\text { rhamnoside }\end{array}$ \\
\hline 18 & 54.06 & $\mathrm{C}_{27} \mathrm{H}_{30} \mathrm{O}_{14}$ & 577.1563 & $\begin{array}{c}577.1558 \\
(30.57)\end{array}$ & 0.83 & 35 & $\begin{array}{c}473.1122(86), \\
431.1010(7), \\
413.0906(31), \\
357.0649(46), \\
339.0550(15), \\
327.0524(35), \\
309.0425(27), \\
298.0508(100), \\
285.0421(3)\end{array}$ & $\begin{array}{l}\text { Luteolin-6-C- } \\
\text { rhamnosyl-2"-O- } \\
\text { rhamnoside }\end{array}$ \\
\hline 19 & 58.56 & $\mathrm{C}_{23} \mathrm{H}_{22} \mathrm{O}_{11}$ & 473.1089 & $\begin{array}{c}473.1122 \\
(82.99)\end{array}$ & -6.68 & 25 & $\begin{array}{c}327.0525(54), \\
311.0593(2), \\
298.0502(100), \\
285.0419(5)\end{array}$ & $\begin{array}{l}\text { Luteolin-6-vinyl-2" - } \\
\text { O-rhamnoside }\end{array}$ \\
\hline 20 & 59.13 & $\mathrm{C}_{27} \mathrm{H}_{28} \mathrm{O}_{13}$ & 559.1457 & $\begin{array}{l}559.1454 \\
(12.12)\end{array}$ & 0.56 & 25 & $\begin{array}{c}457.1091(2), \\
413.0858(2), \\
395.0759(100), \\
351.0838(7), \\
321.0398(32) \\
269.0440(13)\end{array}$ & $\begin{array}{c}\text { Cassiaoccidentalin A } \\
\text { isomer }\end{array}$ \\
\hline 21 & 61.67 & $\mathrm{C}_{27} \mathrm{H}_{28} \mathrm{O}_{13}$ & 559.1457 & $\begin{array}{c}559.1439 \\
(41.19)\end{array}$ & 3.24 & 25 & $\begin{array}{c}457.1090(6), \\
413.0784(3), \\
395.0759(100), \\
351.0867(6) \\
321.0381(30) \\
269.0430(27)\end{array}$ & Cassiaoccidentalin A \\
\hline 22 & 64.71 & $\mathrm{C}_{27} \mathrm{H}_{30} \mathrm{O}_{13}$ & 561.1614 & $\begin{array}{c}561.1612 \\
(23.94)\end{array}$ & 0.29 & 35 & $\begin{array}{c}415.0986(3), \\
397.0923(87), \\
341.0640(25), \\
323.0547(16), \\
311.0555(14) \\
293.0452(93) \\
269.0448(4)\end{array}$ & $\begin{array}{l}\text { Apigenin-6-C- } \\
\text { rhamnosyl-2"-O- } \\
\text { rhamnoside }\end{array}$ \\
\hline
\end{tabular}

a Campare with a reference standards.

were shown to be generated through cross-ring cleavages of the glycoside moiety $[\mathrm{M}-\mathrm{H}-(60 / 90 / 120)]^{-}$(C-arabinoside) and $[\mathrm{M}-\mathrm{H}-(90 / 120 / 150)]^{-}$(C-glucoside). Compound 17 $\left(\mathrm{C}_{21} \mathrm{H}_{20} \mathrm{O}_{10}\right)$ produced a deprotonated ion $[\mathrm{M}-\mathrm{H}]^{-}$at $\mathrm{m} / \mathrm{z}$ 431.0988. The MS/MS spectrum showed three high intensity fragments at $\mathrm{m} / \mathrm{z} 357.0619\left({ }^{0,3} \mathrm{X}_{0}\right)[\mathrm{M}-\mathrm{H}-74]^{-}, 327.0515$ $\left({ }^{0,2} \mathrm{X}_{0}\right)[\mathrm{M}-\mathrm{H}-104]^{-}$and $297.0393\left({ }^{0,1} \mathrm{X}_{0}\right) \quad[\mathrm{M}-\mathrm{H}-134]^{-}$, suggesting the cross-ring cleavage of the rhamnosyl part of the flavonoid glycosyl moiety. The pattern of sugar crossrings of $C$-rhamnose was $[\mathrm{M}-\mathrm{H}-(74 / 104 / 134)]^{-}[29]$. The characteristic product ions at $\mathrm{m} / \mathrm{z} 285.0398\left[\mathrm{Y}_{0}\right]^{-}$, obtained with $\mathrm{CE}=25 \mathrm{eV}$, led to the identification of the aglycone as luteolin. The abundant fragment ion at $m / z 413.0883$ [M-
$\mathrm{H}-18]^{-}$indicates that a rhamnosyl moiety was at the 6-C position. According to the mass spectrometry cleavage pathway of the flavonoid $C$-glycoside standard, compound $17(\mathrm{RT}=50.61 \mathrm{~min})$ was tentatively identified as luteolin-6C-rhamnoside.

\section{Identification of $\mathbf{0}$-glycosides}

Compound $8\left(\mathrm{C}_{21} \mathrm{H}_{20} \mathrm{O}_{11}\right)$ eluted at $24.78 \mathrm{~min}$ and was positively identified as luteolin-7-O-glucoside by comparison of its elution time and mass spectral data with those of authentic standards. The MS/MS spectrum of luteolin-7-Oglucoside yielded a deprotonated ion $[\mathrm{M}-\mathrm{H}]^{-}$at $\mathrm{m} / \mathrm{z}$ 


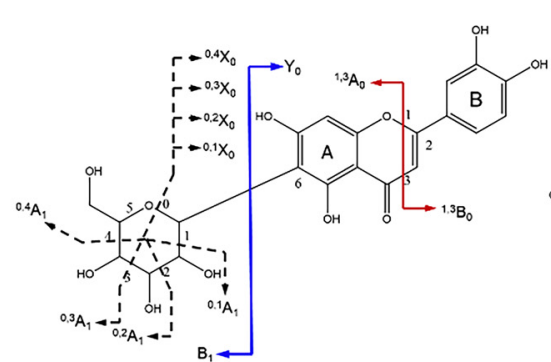

(a)

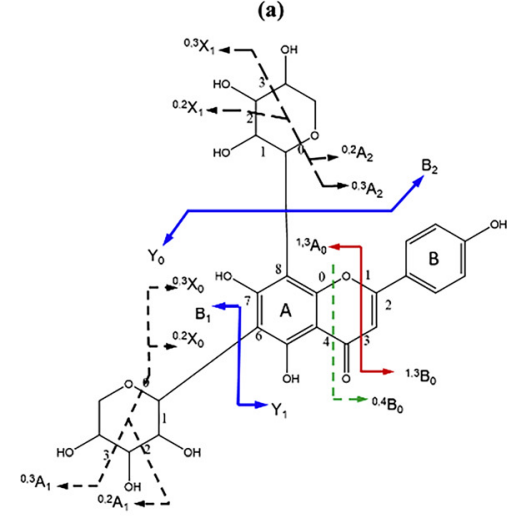

(d)

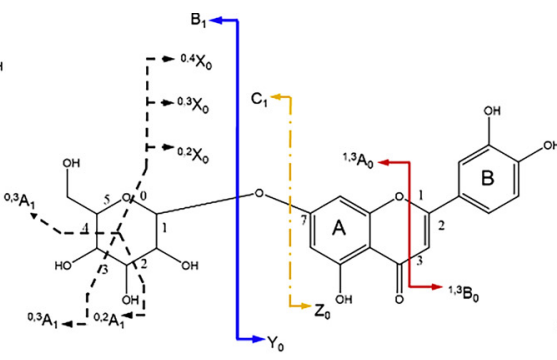

(b)

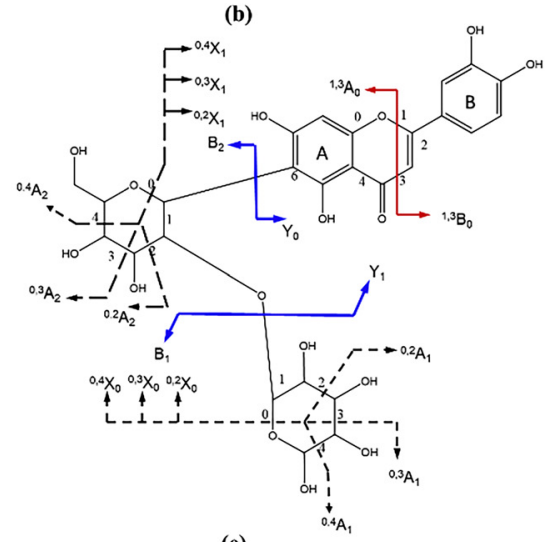

(e)

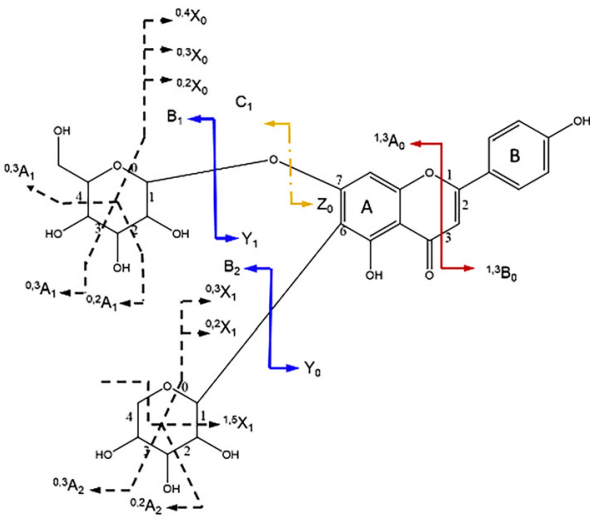

(c)

Scheme 1. Fragmentation nomenclature commonly used for flavonoids (a. luteolin-6-C-glucoside; b. luteolin-7-O-glucoside; c. apigenin-6$C$-arabinosyl-7-O-glucoside; d. 6,8-di-C-arabinoside; and e. luteolin-6-C-glucosyl-2" -O-rhamnoside) according to Domon and Costello [27] and $\mathrm{Ma}$ et al. [28]

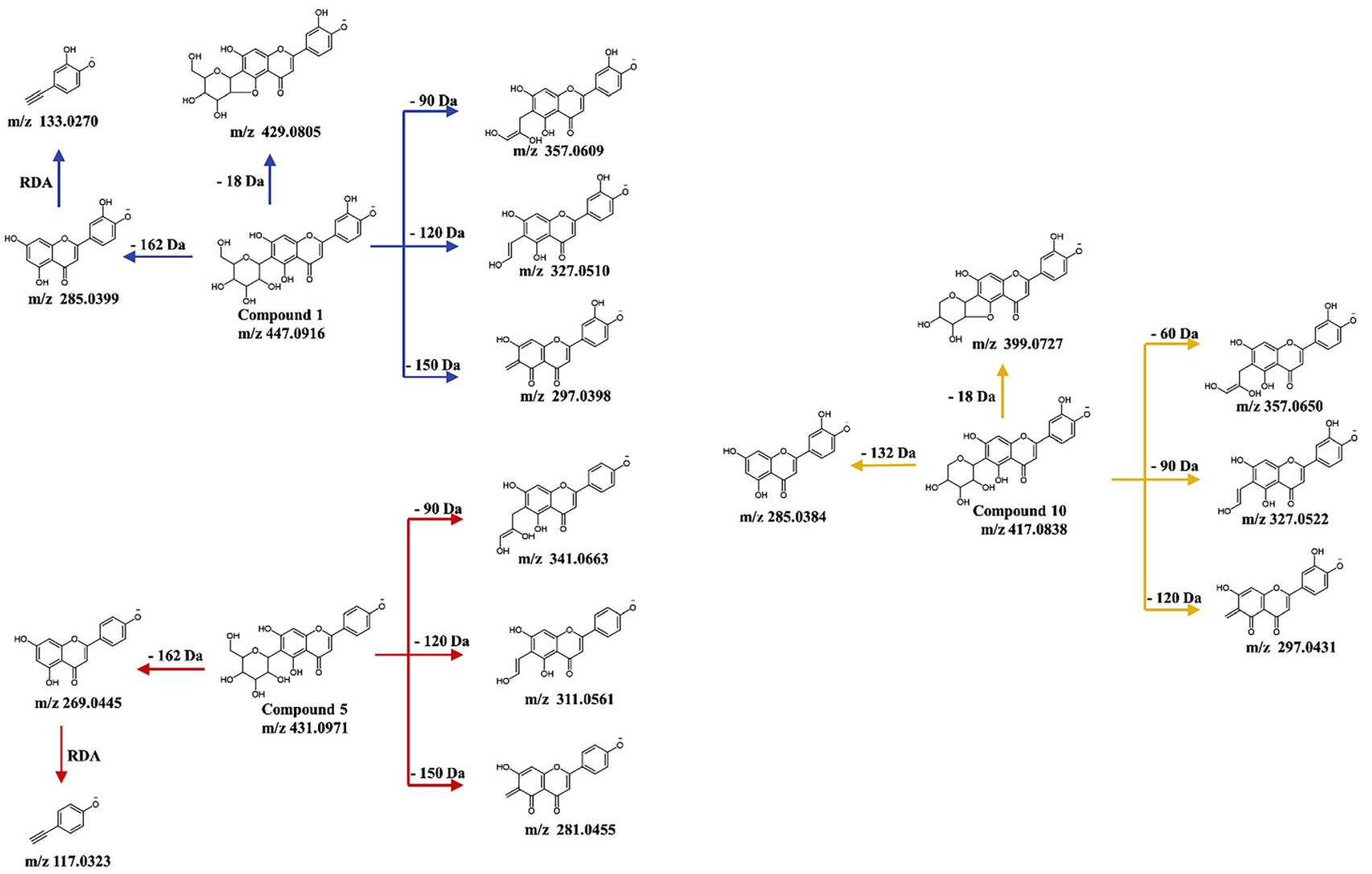

Scheme 2. The main fragmentation pathways of the flavonoid $C$-glycosides (compounds 1,5 and 10) 

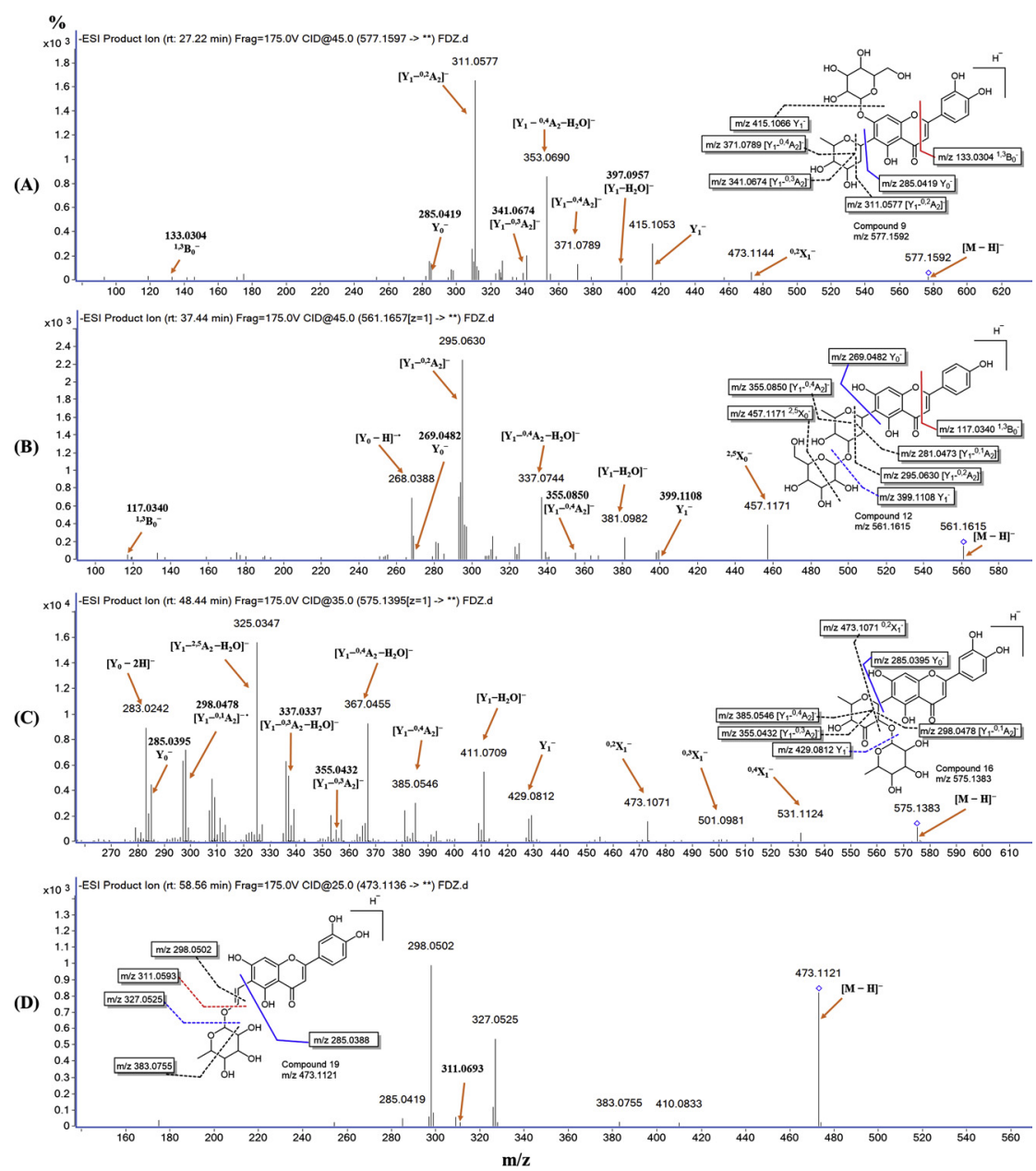

Fig. 3. Product ion spectra and the main fragmentation pathways of the deprotonated ions $[\mathrm{M}-\mathrm{H}]^{-}$of compounds $9,12,16$ and 19 in (-) ESI-MS/MS: (A) compound 9, (B) compound 12, (C) compound 16 and (D) compound 19

447.0953 and showed a fragment ion at $\mathrm{m} / z 285.0423\left[\mathrm{Y}_{0}\right]^{-}$ due to the loss of a glucose, and this ion showed the highest relative abundance. In addition, Table 1 shows that the elimination of $163 \mathrm{Da}$ from the $O$-position produced a fragment ion at $m / z 284.0338\left[\mathrm{Y}_{0}-\mathrm{H}\right]^{-\bullet}$, and its relative intensity was $68 \%$, which indicated that compound 8 was an $\mathrm{O}$-glucoside derivative of luteolin. The $\left[\mathrm{Y}_{0}-\mathrm{H}\right]^{-\bullet}$ ion was typically observed upon cleavage of the glycosidic bond between the aglycone part and the $O$-position that was glycosylated position, and the nature has a significant influence on the fragmentation behaviour of flavonoid $O$ glycosides, especially in flavonoid 3-O- or 7-O-glycosides, and affects the relative abundances of radical aglycone ions $[24,30]$. The fragment ions at $m / z 151.0064\left[{ }^{1,3} \mathrm{~A}_{0}\right]^{-}$and $133.0291\left[{ }^{1,3} \mathrm{~B}_{0}\right]^{-}$were produced by RDA reactions.

The fragmentation pathway of the flavonoid O-glycosides mainly involves losing glycoside moieties from the deprotonated ion through a dehydration condensation reaction that forms high-abundance flavonoid cores and RDA reactions. According to the obtained mass spectrometry data, compound 19 ( $\mathrm{RT}=58.56 \mathrm{~min})$ gave a deprotonated molecular ion $[\mathrm{M}-\mathrm{H}]^{-}$at $m / z$ 473.1122. The $\mathrm{MS}^{2}$ spectrum of compound 19 showed a highly abundant $[\mathrm{M}-\mathrm{H}-146]^{-}$ ion at $m / z 327.0525$, which showed that a rhamnose was attached to the $O$-position. The fragmentation ion at $\mathrm{m} / z$ 285.0419 indicated that compound 19 was a derivative of luteolin. The characteristic ions at $\mathrm{m} / z \quad 311.0593$ and 298.0502 were produced by the losses of 26 and $13 \mathrm{Da}$ from the product ion at $m / z 285.0419$, which indicated that a vinyl was attached to the $C$-position of the flavonoid and a rhamnose. Therefore, it was tentatively identified as luteolin6-vinyl-2"-O-rhamnoside. Product ion spectra and the main fragmentation pathways of the deprotonated ion $[\mathrm{M}-\mathrm{H}]^{-}$ of compound 19 in (-)ESI MS/MS are shown in Fig. 3.

\section{Identification of di-C,0-glycosides}

The RT of compound $4\left(\mathrm{C}_{26} \mathrm{H}_{28} \mathrm{O}_{14}\right)$ was $18.53 \mathrm{~min}$, and it was confidently assigned as apigenin-6-C-arabinosyl-7-Oglucoside by comparing its RT and MS/MS fragmentation pattern with those of the reference standard (Scheme 3). A series of characteristic product ions were observed in the $\mathrm{MS}^{2}$ spectra of the $[\mathrm{M}-\mathrm{H}]^{-}$ions at $\mathrm{m} / z 563.1390$ for compound 4. The MS ${ }^{2}$ data in Table 1 showed an abundant fragment ion at $m / z 401.0837\left[\mathrm{Y}_{1}\right]^{-}$generated by the loss of the glycoside moiety from the deprotonated molecular ion, 


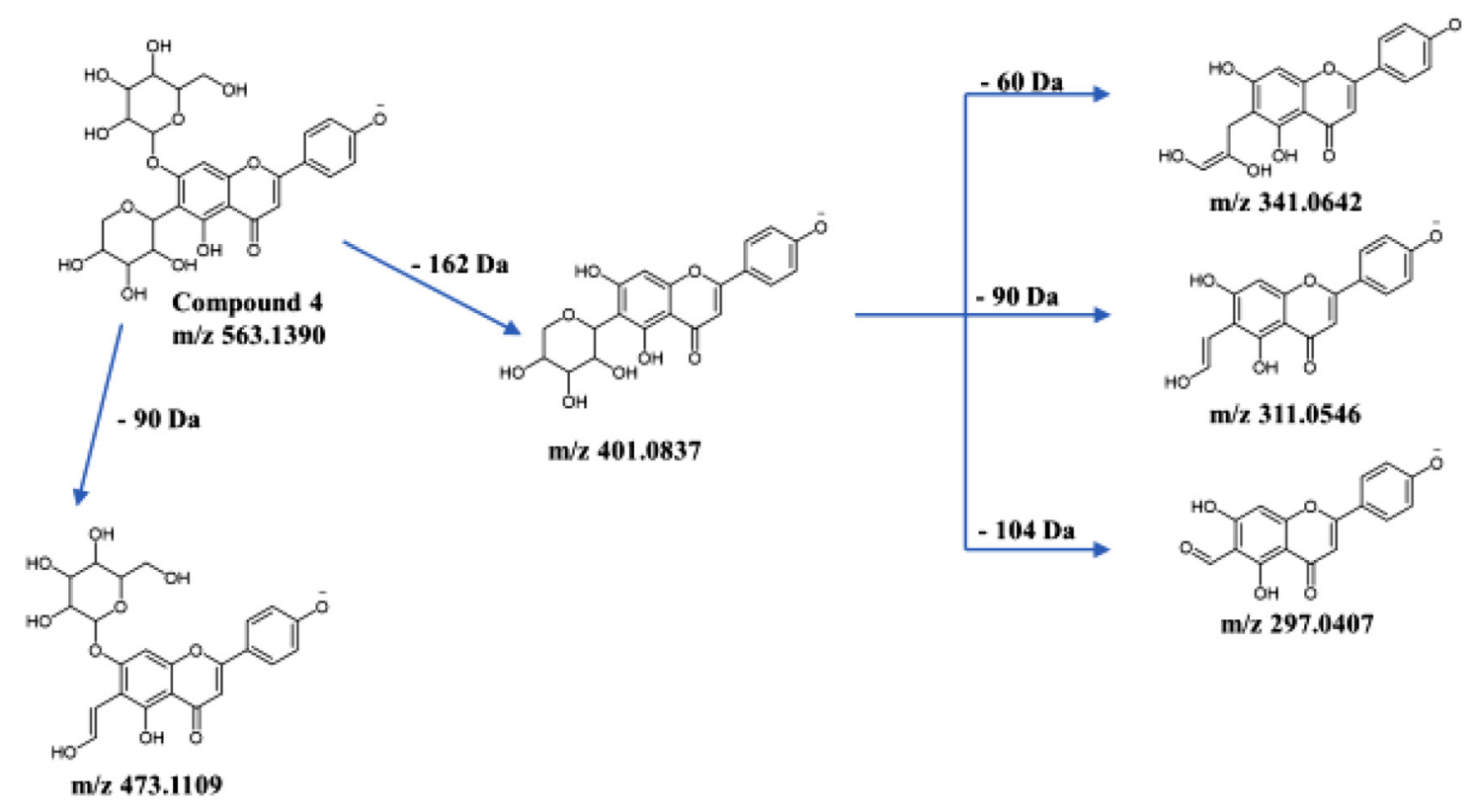

Scheme 3.. The main fragmentation pathways of the flavonoid di-C,O-glycosides (compound 4)

which indicated the presence of an O-glucosyl unit. The fragmentation pathways of the fragment ions at $\mathrm{m} / \mathrm{z}$ $473.1109\left({ }^{0,2} \mathrm{X}_{1}\right) \quad[\mathrm{M}-\mathrm{H}-90]^{-}, \quad 341.0642 \quad\left[\mathrm{Y}_{1}-60\right]^{-}$, $311.0546\left[\mathrm{Y}_{1}-90\right]^{-}$and $297.0407\left[\mathrm{Y}_{1}-120\right]^{-}$indicated the presence of a $C$-arabinose unit and an apigenin flavonoid core.

As stated above, the main fragmentation pathways of the flavonoid di-C,O-glycosides in (-)ESI-MS/MS involved the direct loss of glycoside moieties from the $O$-position through a dehydration condensation reaction, forming high-abundance characteristic ions and the cross-ring cleavage of the aglycone moiety at the $C$-position. As shown in Table 1, compounds $3(\mathrm{RT}=18.47 \mathrm{~min})$ and $9(\mathrm{RT}=27.22 \mathrm{~min})$ showed deprotonated molecular ions at $\mathrm{m} / z 593.1501$ and 577.1592 , respectively, both of which have abundant fragment ions from the loss of a glycoside moiety from the deprotonated molecular ions at $\mathrm{m} / z 431.1003\left[\mathrm{Y}_{1}\right]^{-}$and $415.1053\left[\mathrm{Y}_{1}\right]^{-}$, respectively, indicating that glucose is in the $O$-position of the aglycone. The fragment ions at $\mathrm{m} / \mathrm{z}$ $285.0442\left[\mathrm{Y}_{0}\right]^{-}$and $285.0419\left[\mathrm{Y}_{0}\right]^{-}$indicated that these compounds are luteolin derivatives. Furthermore, a series of other characteristic product ions at $\mathrm{m} / z 357.0636\left[\mathrm{Y}_{1}-74\right]^{-}$ and $327.0550\left[\mathrm{Y}_{1}-104\right]^{-}$as well as $\mathrm{m} / z$ 473.1144 $\left({ }^{1,5} \mathrm{X}_{1}\right)$ $[\mathrm{M}-\mathrm{H}-104]^{-}, 371.0789\left[\mathrm{Y}_{1}-44\right]^{-}, 341.0674\left[\mathrm{Y}_{1}-74\right]^{-}$and $311.0577\left[\mathrm{Y}_{1}-104\right]^{-}$, as shown in Table 1 , signified that these compounds had rhamnosyl and boivinosyl moieties at their $C$-positions, respectively. The pattern of sugar crossrings of $\mathrm{C}$-boivinose was $[\mathrm{M}-\mathrm{H}-(44 / 74 / 104)]^{-}$. Thus, compounds $3\left(\mathrm{C}_{27} \mathrm{H}_{30} \mathrm{O}_{16}\right)$ and $9\left(\mathrm{C}_{27} \mathrm{H}_{30} \mathrm{O}_{14}\right)$ were tentatively assigned as luteolin-8-C-rhamnosyl-4'-O-glucoside and luteolin- $\mathrm{C}$-boivinosyl-O-glucoside according to the fragmentation pathway of flavonoid $\mathrm{C}, \mathrm{O}$-di-glycosides. Product ion spectra and the main fragmentation pathways of the deprotonated ion $[\mathrm{M}-\mathrm{H}]^{-}$of compound 9 in $(-) \mathrm{ESI}$ MS/MS are shown in Fig. 3.

\section{Identification of di-C-glycosides}

Compound $7(\mathrm{RT}=24.57 \mathrm{~min})$ has an $[\mathrm{M}-\mathrm{H}]^{-}$ion at $\mathrm{m} / z$ 533.1285, which suggests a formula of $\mathrm{C}_{25} \mathrm{H}_{26} \mathrm{O}_{13}$, and comparison of its elution time and tandem mass spectrum with those of the reference standard indicated that compound 7 was apigenin 6,8-di- $C$-arabinoside. The tandem mass spectrum of compound 7 contained major product ions at $m / z \quad 473.1077\left({ }^{0,3} \mathrm{X}_{1}\right.$ or $\left.{ }^{0,3} \mathrm{X}_{2}\right) \quad[\mathrm{M}-\mathrm{H}-60]^{-}$and $443.0977\left({ }^{0,2} \mathrm{X}_{1}\right.$ or $\left.{ }^{0,2} \mathrm{X}_{2}\right)[\mathrm{M}-\mathrm{H}-90]^{-}$, consistent with the fragmentation pattern of flavonoid di-C-glycosides, and indicating the losses of 60 and $90 \mathrm{Da}$ from the $[\mathrm{M}-\mathrm{H}]^{-}$ion via the cross-ring cleavage of the arabinoside part of the flavonoid glycosyl moiety. The ions at $\mathrm{m} / z$ 413.0856 [M$\mathrm{H}-(60+60)]^{-}, 383.0776[\mathrm{M}-\mathrm{H}-(90+60)]^{-}$and 353.0657 $[\mathrm{M}-\mathrm{H}-(90+90)]^{-}$indicated the presence of apigenin as the aglycone with two pentose substituents (arabinose). Among them, the most prominent ion was at $\mathrm{m} / z$ 383.0776, which is most likely because apigenin 6,8 -di- $C$-arabinoside can produce this ion through two cleavage pathways; either the 6- $C$ arabinoside loses $60 \mathrm{Da}$ and the 8-C arabinose loses $90 \mathrm{Da}$ or vice versa. Furthermore, the fragment ions at $\mathrm{m} / z 269.0418$ $\left[\mathrm{Y}_{0}\right]^{-}$, indicated that the flavonoid core was apigenin. The fragmentation scheme of compound 7 is shown in Scheme 4.

\section{Identification of C,0-di-glucosides}

Compounds $2(\mathrm{RT}=15.87 \mathrm{~min})$ and $14(\mathrm{RT}=44.77 \mathrm{~min})$ were positively identified as luteolin-6- $C$-glucosyl- $2^{\prime \prime}-O$ rhamnoside and apigenin-6- $C$-arabinosyl- $2^{\prime \prime}-O$-rhamnoside, respectively, by comparison of their RTs and mass spectral analysis with the authentic reference standards. Compound 2 produced a deprotonated molecular ion at $\mathrm{m} / z 593.1514$ with the chemical formula $\mathrm{C}_{27} \mathrm{H}_{30} \mathrm{O}_{15}$. Compound 2 produced ions corresponding to the loss of the glycoside moiety at $\mathrm{m} / \mathrm{z} 285.0403\left[\mathrm{Y}_{0}\right]^{-}$, which indicated that 2 was a 


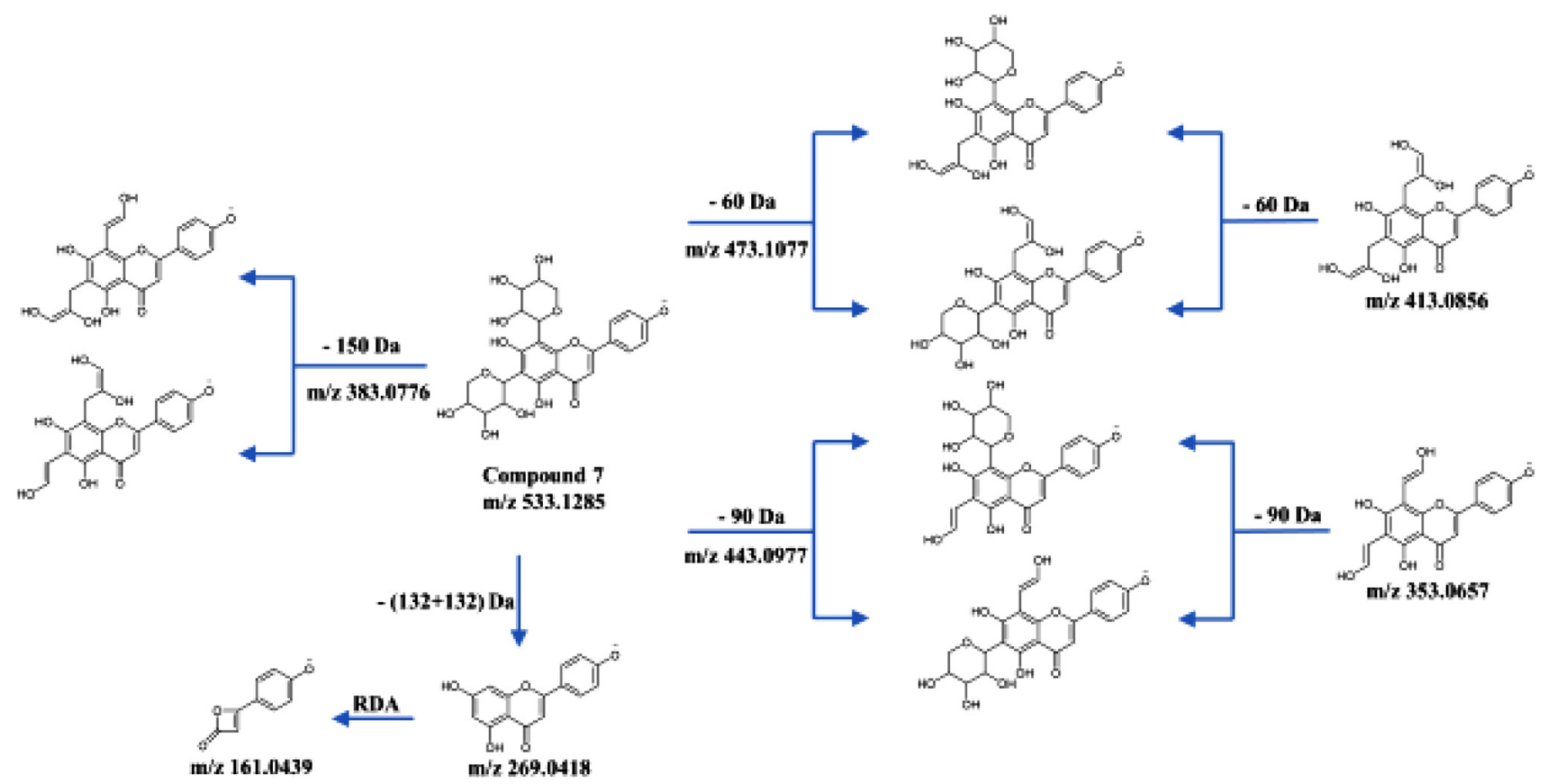

Scheme 4.. The main fragmentation pathways of the flavonoid di-C-glycosides (compound 7)

derivative of luteolin. The fragment ion of compound 2 at $m / z 429.0841\left[\mathrm{Y}_{1}-\mathrm{H}_{2} \mathrm{O}\right]^{-}$was produced by the loss of the terminal sugar plus water, indicating that the rhamnose was attached to the glycoside. The loss of $120 \mathrm{Da}$ to yield the ion at $m / z 473.1106\left({ }^{0,2} \mathrm{X}_{1}\right)$, produced by the internal cleavage of the glucosyl linkage to the aglycone, confirmed that the substitution is not on the 6- or 8-position of that sugar and confirmed the interglycosidic linkage, rhamnosyl $(1 \rightarrow 2)$ glucoside. Furthermore, the fragment ions at $357.0632\left[\mathrm{Y}_{1}-\right.$ $90]^{-}$and $327.0514\left[\mathrm{Y}_{1}-120\right]^{-}$were characteristic of the cross-ring cleavage of the glucose part of the flavonoid glycosyl moiety, and the characteristic product ions at $\mathrm{m} / \mathrm{z}$ 339.0516 and 309.0422 were generated by the loss of $18 \mathrm{Da}$ $\left(\mathrm{H}_{2} \mathrm{O}\right)$ from 357.0632 to 327.0514 , respectively. Compound $14\left(\mathrm{C}_{26} \mathrm{H}_{28} \mathrm{O}_{13}\right)$ yielded a deprotonated molecular ion at $\mathrm{m} / \mathrm{z}$ 547.1439 and produced a flavonoid core at $\mathrm{m} / z 269.0446$ $\left[\mathrm{Y}_{0}\right]^{-}$by losing a glycoside moiety, which indicated that 14 was a derivative of apigenin. Similarly, compound 14 lost 146 and $164 \mathrm{Da}$ to produce the ions at $\mathrm{m} / z 401.0877\left[\mathrm{Y}_{1}\right]^{-}$ and $383.0763\left[\mathrm{Y}_{1}-\mathrm{H}_{2} \mathrm{O}\right]^{-}$, which indicated that it was a $\mathrm{C}, \mathrm{O}-$ di-glucoside and rhamnose was attached to the glycoside. The ions at $\mathrm{m} / \mathrm{z} 457.1140\left({ }^{0,2} \mathrm{X}_{1}\right) \quad[\mathrm{M}-\mathrm{H}-90]^{-}, \mathrm{m} / \mathrm{z}$ $341.0659\left[\mathrm{Y}_{1}-60\right]^{-}$and $311.0553\left[\mathrm{Y}_{1}-90\right]^{-}$were produced from $[\mathrm{M}-\mathrm{H}]^{-}$and $\left[\mathrm{Y}_{1}\right]^{-}$, respectively, via the cross-ring cleavage of the arabinose part of the flavonoid glycosyl moiety. In addition, the fragment ions at $\mathrm{m} / \mathrm{z} 323.0548$ and 293.0443 were generated by the loss of $18 \mathrm{Da}\left(\mathrm{H}_{2} \mathrm{O}\right)$ from the ions at $\mathrm{m} / \mathrm{z} 341.0659$ and 311.0553 . The fragmentation schemes of compounds 2 and 14 are shown in Scheme 5.

The $\mathrm{MS}^{2}$ fragmentation patterns of all of these compounds showed ions produced by the loss of a terminal sugar plus water: (compound $12\left[\mathrm{Y}_{1}-\mathrm{H}_{2} \mathrm{O}\right]^{-}$and compounds $6,11,13,15,16,18,20,21$ and $\left.22\left[\mathrm{Y}_{1}-\mathrm{H}_{2} \mathrm{O}\right]^{-}\right)$, and the loss of a glycosyl residue ( -162 , glucosyl; -146 , rhamnosyl) was also observed, indicating an interglycosidic linkage and suggesting that these ten compounds were $\mathrm{C}, \mathrm{O}$ di-glucosides. Compound 6 showed a deprotonated [M$\mathrm{H}]^{-}$ion at $\mathrm{m} / \mathrm{z} 577.1553$, a product ion at $\mathrm{m} / \mathrm{z} 269.0401$ $\left[\mathrm{Y}_{0}\right]^{-}$, and RDA fragment ions at $\mathrm{m} / \mathrm{z} 117.0309\left[{ }^{1,3} \mathrm{~B}_{0}\right]^{-}$ (obtained with $\mathrm{CE}=35 \mathrm{eV}$ ), which led to the aglycone being identified as apigenin. The fragmentation ion at $\mathrm{m} / \mathrm{z}$ $457.1134\left({ }^{0,2} \mathrm{X}_{1}\right)[\mathrm{M}-\mathrm{H}-120]^{-}$was produced by the crossring cleavage of the glucose part of the flavonoid glycosyl moiety, and $m / z 431.1001\left[\mathrm{Y}_{1}\right]^{-}$and $413.0873\left[\mathrm{Y}_{1}-\mathrm{H}_{2} \mathrm{O}\right]^{-}$ were produced by the loss of the terminal rhamnosyl moiety and the loss of the terminal rhamnosyl moiety plus water, respectively. Furthermore, the fragmentation ions at $\mathrm{m} / \mathrm{z}$ $341.0658\left[\mathrm{Y}_{1}-90\right]^{-}$and $311.0561\left[\mathrm{Y}_{1}-120\right]^{-}$were produced by the cross-ring cleavage of the glucose part of the flavonoid glycosyl moiety, and those at $353.0671\left[\mathrm{Y}_{1}-\mathrm{H}_{2} \mathrm{O}-\right.$ $60]^{-}, 323.0552\left[\mathrm{Y}_{1}-\mathrm{H}_{2} \mathrm{O}-90\right]^{-}$, and $293.0452\left[\mathrm{Y}_{1}-\mathrm{H}_{2} \mathrm{O}-\right.$ $120]^{-}$were produced by the cross-ring cleavage of the ion at $\mathrm{m} / \mathrm{z}$ 413.0873. The characteristic product ions of compound $6(\mathrm{RT}=23.2 \mathrm{~min})$ were $16 \mathrm{Da}$ lighter than those of compound 2 due to the presence of an apigenin flavonoid instead of luteolin; thus, it was tentatively identified as apigenin-6$\mathrm{C}$-glucosyl-2" -O-rhamnoside by comparison with reference standards and the literature data [31]. Similarly, compound $11(\mathrm{RT}=31.92 \mathrm{~min})$ showed an $[\mathrm{M}-\mathrm{H}]^{-}$ion at $\mathrm{m} / \mathrm{z}$ 563.1416 and produced prominent ions at $\mathrm{m} / \mathrm{z} 285.0398$ $\left[\mathrm{Y}_{0}\right]^{-}$, which indicated that 11 was a derivative of luteolin. The characteristic product ions of compound 11 were $16 \mathrm{Da}$ heavier than those of compound 14 because of the luteolin flavonoid core. Hence, 11 was tentatively identified as luteolin-6- $C$-arabinosyl-2" ${ }^{\prime \prime}$-O-rhamnoside [32]. Compounds $13(\mathrm{RT}=39.11 \mathrm{~min})$ and $18(\mathrm{RT}=54.06 \mathrm{~min})$ showed deprotonated molecular ions at $\mathrm{m} / z$ 577.1587 and 577.1558, respectively, indicating that they were isomers. Compounds 


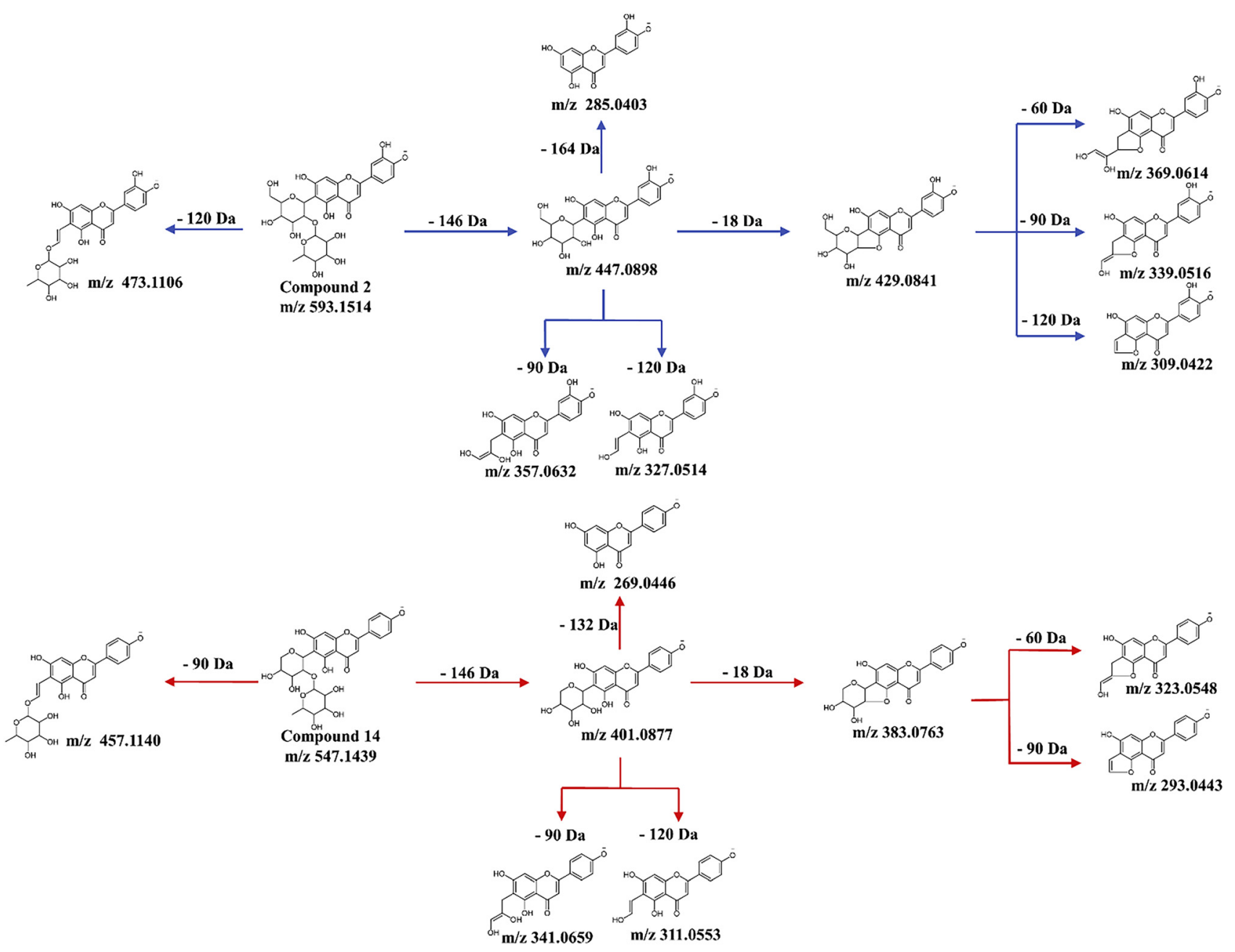

Scheme 5.. The main fragmentation pathways of the flavonoid C,O-di-glycosides (compound 2 and compound 14)

13 and 18 produced ions corresponding to the loss of a glycoside moiety at $\mathrm{m} / \mathrm{z} 285.0432\left[\mathrm{Y}_{0}\right]^{-}$, which indicated that 13 and 18 were derivatives of luteolin. Based on their mass spectral data and the characteristic product ions at $\mathrm{m} / \mathrm{z}$ $357.0638\left[\mathrm{Y}_{1}-74\right]^{-}, 339.0505\left[\mathrm{Y}_{1}-\mathrm{H}_{2} \mathrm{O}-74\right]^{-}, 327.0521$ $\left[\mathrm{Y}_{1}-104\right]^{-}$and $309.0383\left[\mathrm{Y}_{1}-\mathrm{H}_{2} \mathrm{O}-104\right]^{-}$, obtained at $\mathrm{CE}$ $=35 \mathrm{eV}$, compounds 13 and 18 were tentatively identified as luteolin-C-rhamnosyl-2" -O-rhamnoside isomers. Compound $12(\mathrm{RT}=37.44 \mathrm{~min})$ showed a deprotonated $[\mathrm{M}-$ $\mathrm{H}]^{-}$ion at $\mathrm{m} / z 561.1615$ and produced an ion at $\mathrm{m} / \mathrm{z}$ $269.0482\left[\mathrm{Y}_{0}\right]^{-}$as well as an RDA fragment at $m / z 117.0340$ $\left[{ }^{1,3} \mathrm{~B}_{0}\right]^{-}$, which indicated an apigenin core of flavonoid. Furthermore, the fragmentation ions at $m / z 457.1171\left({ }^{2,5} \mathrm{X}_{0}\right)$ $[\mathrm{M}-\mathrm{H}-104]^{-}$, $399.1108\left[\mathrm{Y}_{1}\right]^{-}$and $381.0928\left[\mathrm{Y}_{1}-\mathrm{H}_{2} \mathrm{O}\right]^{-}$ indicated a hexose linked at the $C$-boivonosyl moiety. A series of characteristic product ions at $\mathrm{m} / z 355.0850$ [ $\mathrm{Y}_{1}-$ $44]^{-}, 295.0630\left[\mathrm{Y}_{1}-104\right]^{-}$and $281.0473\left[\mathrm{Y}_{1}-118\right]^{-}$were observed in the $\mathrm{MS}^{2}$ spectra and showed the pattern of sugar cross-rings of C-boivonose [M-H-(44/74/104/118) $]^{-}$. Hence, compound 12 was tentatively identified as apigenin6-C-boivonosyl-3" $-O$-glucoside. Product ion spectra and the main fragmentation pathways of the deprotonated ion [M$\mathrm{H}]^{-}$of compound 12 in (-)ESI MS/MS are shown in Fig. 3.
Similarly, compound $22(\mathrm{RT}=64.71 \mathrm{~min})$ produced a $[\mathrm{M}-$ $\mathrm{H}]^{-}$ion at $m / z 561.1612$ and produced a fragment ion at $\mathrm{m} / z$ $269.0448\left[\mathrm{Y}_{0}\right]^{-}$, which indicated that 22 was a derivative of apigenin. The characteristic product molecules of compound 22 were 16 Da lighter than those of compounds 13 and 18 because of the apigenin flavonoid core. Therefore, compound 22 was tentatively identified as apigenin-6- $C$-rhamnosyl-2" $-O$-rhamnoside. Compounds $15(\mathrm{RT}=45.54 \mathrm{~min})$ and $16(\mathrm{RT}=48.44 \mathrm{~min})$ gave the $[\mathrm{M}-\mathrm{H}]^{-}$ions at $\mathrm{m} / z$ 575.1397 and 575.1383. The MS-MS spectrum of compound 16 showed three obvious fragmentation ions at $\mathrm{m} / z 429.0812$ $\left[\mathrm{Y}_{1}\right]^{-}, 411.0709\left[\mathrm{Y}_{1}-\mathrm{H}_{2} \mathrm{O}\right]^{-}$and $285.0395\left[\mathrm{Y}_{0}\right]^{-}$, indicating that a pentose was linked at the $C$-dehydropentanoside. The characteristic fragmentation ions at $473.1072\left({ }^{0,2} \mathrm{X}_{1}\right)[\mathrm{M}-$ $\mathrm{H}-102]^{-}, 385.0546\left[\mathrm{Y}_{1}-44\right]^{-}$and $298.0478\left[\mathrm{Y}_{1}-131\right]^{-\bullet}$ showed a $C$-dehydropentanoside, and which indicated that dehydrogenation occurred at the hydroxy group at the $3^{\prime}$ position of pentose. In addition, compound 16 showed MS/ MS spectra similar to those of compound 15. Based on their tandem mass spectra and the literature data [33], compounds 15 and 16 were tentatively identified as isomers of cassiaoccidentalin B. Product ion spectra and the main fragmentation pathways of the deprotonated ion $[\mathrm{M}-\mathrm{H}]^{-}$ 
of compound 16 in (-)ESI MS/MS are shown in Fig. 3. Compound $20(\mathrm{RT}=59.13 \mathrm{~min})$ showed an $[\mathrm{M}-\mathrm{H}]^{-}$ion at $\mathrm{m} / z 559.1454$ and fragment ions at $\mathrm{m} / z 413.0858\left[\mathrm{Y}_{1}\right]^{-}$, $395.0759\left[\mathrm{Y}_{1}-\mathrm{H}_{2} \mathrm{O}\right]^{-}$and $269.0440\left[\mathrm{Y}_{0}\right]^{-}$, which indicated that 20 was a $C, O$-di-glycoside derivative of apigenin. The product ions at $\mathrm{m} / \mathrm{z} 457.1091 \quad\left({ }^{0,2} \mathrm{X}_{1}\right) \quad[\mathrm{M}-\mathrm{H}-102]^{-}$, $\left.351.0838\left[\mathrm{Y}_{1}-\mathrm{H}_{2} \mathrm{O}-44\right)\right]^{-}$and $321.0398\left[\mathrm{Y}_{1}-\mathrm{H}_{2} \mathrm{O}-74\right]^{-}$ showed a $C$-dehydropentanoside, which indicated dehydrogenation occurred at the hydroxy group at the $3^{\prime}$-position of pentose. In addition, compound 21 showed MS/MS spectra similar to those of compound 20, which showed they were isomers. The fragmentation ions of compounds 20 and 21 were 16 Da lighter than those of compounds 15 and 16 based on the apigenin core of compounds 20 and 21, and they were tentatively identified as isomers of cassiaoccidentalin A.

\section{Analysis of the occurrence of these flavonoids in other bamboo species}

Compared with the data found in the literature, a total of ten of these flavonoids have been identified or isolated from other bamboo species, and the other 12 flavonoids have not been found in other bamboo leaves. Compounds 1 and 5 were widely reported in many species bamboo leaves of many genera, such as Bambusa, Phyllostachys, Sasa, Pleioblastus, Dendrocalamopsis, Dendrocalamus and Pseudosasa [34-40]. Compound 2 was identified from the leaves of Bambusa textilis, Sasa borealis and Pleioblastus amarus [4143]. Compounds 4 and 6 were both identified in the leaves of B. textilis $[36,41]$. Compound 8 was found in the leaves of many bamboo species, such as $B$. textilis, Dendrocalamopsis oldhami $[40,41]$. Besides, compounds 9 and 10 were isolated from the leaves of Fargesia robusta and Bambusa pervariabilis, respectively, and compound 10 was tentatively identified from B. textilis and Pseudosasa japonica [34, 44, 45]. Compound 15 was tentatively identified from the leaves of nine species of bamboo [34]. Compound 19 was isolated in the leaves of $P$. amarus [43].

\section{CONCLUSION}

In conclusion, a rapid and accurate UPLC-ESI-Q-TOF-MS/ MS method using the negative ion mode was developed to identify the structures of flavonoids in B. chungii leaves extract. The identities of the flavonoids were determined using the MS/MS spectra of standards of three $C$-glycoside, one $\mathrm{O}$-glycoside, di-C,O-glycoside and di- $\mathrm{C}$-glycoside as well as two $\mathrm{C}, \mathrm{O}$-di-glucoside flavonoids as templates for interpretation. A total of 22 flavonoids, including $4 \mathrm{C}$-glycosides, $2 \mathrm{O}$-glycosides, 3 di-C,O-glycosides, $1 \mathrm{di}$ - $\mathrm{C}$-glycoside and 12 $\mathrm{C}, \mathrm{O}$-di-glucosides, were either identified or tentatively identified in the B. chungii leaves extract based on their MS/ MS behaviour and fragmentation pathways. To the best of our knowledge, this is the first report of the comprehensive analysis of the flavonoid glycosides in $B$. chungii leaves extract by UPLC/ESI-QTOF-MS. The present work demonstrates the efficiency of UPLC-ESI-Q-TOF-MS/MS for identifying multiple flavonoids in BLE and separating and distinguishing isomers present in complex mixtures based on their characteristic fragment ions.

Conflict of interest: The authors declared no potential conflicts of interest with respect to the research, authorship, and/or publication of this article.

\section{ACKNOWLEDGEMENT}

This work was financially supported by National Natural Science Foundation of China (project No. 31570345).

\section{SUPPLEMENTARY MATERIAL}

Supplementary material to this article can be found online at https://doi.org/10.1556/1326.2020.00777.

\section{REFERENCES}

1. Hu, C.; Zhang, Y.; Kitts, D. D. J. Agric. Food Chem. 2000, 48, 3170.

2. Gong, J. Y.; Xia, D. Z.; Huang, J.; Ge, Q.; Mao, J.; Liu, S.; Zhang, Y. J. Med. Food 2015, 18, 453.

3. Luo, G. Y.; Luo, Y. G.; Zhou, R.; Zhou, M.; Gu, J.; Ye, Q.; Dai, Y.; Zhang. G. L. J. Asian. Nat. Prod. Res. 2015, 17, 248.

4. Lu, B.; Wu, X.; Shi, J.; Zhang, Y. Food Chem. Toxicol. 2006, 44, 1739.

5. Gao, Q.; Shi, Y. H.; Liao, M.; Xiao, J. J.; Li, X. X.; Zhou, L. J.; Liu, C. W.; Liu, P.; Cao, H. Q. Pest Manag. Sci. 2019, 75, 3167.

6. Wu, D.; Chen, J. Y.; Lu, B. Y.; Xiong, L.; He, Y.; Zhang, Y. Food Chem. 2012, 135, 2147.

7. Ni, Q. X.; Xu, G. Z.; Wang, Z. Q.; Gao, Q. X.; Wang, S.; Zhang, Y. Z. Int. J. Mol. Sci. 2012, 13, 2249.

8. Wang, B.; Wei, W. J.; Liu, C. J. J. Trop. Forest Sci. 2013, 25, 137.

9. Yang, Y. Y.; Bai, L.; Li, X. R.; Xiong, J.; Xu, P. X.; Guo, C. Y.; Xue, M. Toxicol. in Vitro. 2014, 28, 388.

10. Zhang, Y.; Luo, Z.; Shao, Z.; Yu, C. D.; Wang, S. J. Agric. Food Chem. 2014, 62, 4798.

11. Yu, Y.; Li, Z. M.; Cao, G. T.; Huang, S. D.; Yang, H. S. J. Food Sci. 2019, 84, 1609.

12. Madushani Herath, K. H. I. N.; Cho, J.; Kim, A.; Eom, T. K., Kim, J.-S., Kim, J.-B., Doh, Y. H.; Jee, Y. J. Ethnopharmacol. 2018, 224, 335.

13. Yang, J. P.; He, H.; Lu, Y. H. J. Agric. Food Chem. 2014, 62, 7760.

14. Wedler, J.; Daubitz, T.; Schlotterbeck, G.; Butterweck, V. Planta Med. 2014, 80, 1678.

15. Sun, S. W.; Liu, M. J.; He, J.; Li, K. P.; Zhang, X. G.; Yin, G. L. Molecules 2019, 24, 1791.

16. Lee, Y. H.; Kim, B.; Hwang, S. R.; Kim, K.; Lee J. H. J. Food Drug Anal. 2018, 26, 277.

17. Taamalli, A.; Iswaldi, I.; Arráez-Román, D.; Carretero, A. S.; Fernández-Gutiérrez, A.; Zarrouk, M. Phytochem. Anal. 2014, 25, 89. 
18. Quirantes-Piné, R.; Lozano-Sánchez, J.; Herrero, M.; Ibáñez, E.; SeguraCarretero, A.; Fernández-Gutiérrez, A. Phytochem. Anal. 2013, 24, 213.

19. Yan, M.; Chen, M.; Zhou, F.; Cai, D. S.; Bai, H.; Wang, P. L.; Lei, H. M.; Ma, Q. J. Pharm. Biomed. Anal. 2019, 164, 734.

20. Stobiecki, M. Phytochem. 2000, 54, 237.

21. Shi, P. Y.; He, Q.; Song, Y.; Qu, H. B.; Cheng, Y. Y. Anal. Chim. Acta 2007, 598, 110.

22. Geng, P.; Sun, G. H.; Zhang, M. L.; Li, X. N.; Harnly, J. M.; Chen, P. J. Mass Spectrom. 2016, 51, 914.

23. Gonzales, G. B.; Raes, K.; Coelus, S.; Struijs, K.; Smagghe, G.; Camp, J. V. J. Chromatogr. A 2014, 1323, 39.

24. Singh, A.; Kumar, S.; Bajpai, V.; Reddy, T. J.; Rameshkumar, K. B.; Kumar, B. Rapid Commun. Mass Spectrom. 2015, 29, 1095.

25. Li, Z. H.; Guo, H.; Xu, W. B.; Ge, J.; Li, X.; Alimu, M.; He, D. J. J. Chromatogr. Sci. 2016, 54, 805.

26. Qin, Y.; Gao, B.; Shi, H.; Cao, J.; Yin, C. L.; Lu, W. Y.; Yu, L. L.; Cheng, Z. H. J. Pharm. Biomed. Anal. 2017, 142, 113.

27. Domon, B.; Costello, C. E. Glycoconj. J. 1988, 5, 397.

28. Ma, Y. L.; Li, Q. M.; Heuvel, H. V. D.; Claeys, M. Rapid Commun. Mass Spectrom. 1997, 11, 1357.

29. Vukics, V.; Guttman, A. Mass Spectrom. Rev. 2010, 29, 12.

30. Ablajan, K.; Abliz, Z.; Shang, X. Y.; He, J. M.; Zhang, R. P.; Shi J. G. J. Mass Spectrom. 2006, 41, 352.

31. Sun, Y.; Hui, Q. R.; Chen, R.; Li, H. P.; Peng, H.; Chen, F.; Deng, Z. Y. J. Funct. Foods 2018, 40, 349.
32. Figueirinha, A.; Paranhos, A.; Pérez-Alonso, J. J.; Santos-Buelga, C.; Batista, M. T. Food Chem. 2008, 110, 718.

33. Pérez, A. J.; Hussain, S. M.; Pecio, Ł.; Kowalczyk, M.; Herling, V. R.; Stochmal, A. J. Agric. Food Chem. 2016, 64, 4686.

34. Van Hoyweghen, L.; De Beer, T.; Deforce, D.; Heyerick, A. Phytochem. Anal. 2012, 23, 433.

35. Wang, J.; Yue, Y. D.; Jiang, H.; Tang, F. Int. J. Anal. Chem. 2012, 1, 205101.

36. Wang, J.; Yue, Y. D.; Tang, F.; Sun, J. Molecules 2012, 17, 8872.

37. Zhang, Y.; Jiao, J. J.; Liu, C. M.; Wu, X. Q.; Zhang, Y. Food Chem. 2008, 107, 1326.

38. Ni, Q. X.; Xu, G. Z.; Wang, Z. Q.; Gao, Q. X.; Wang, S.; Zhang, Y. Z. Int. J. Mol. Sci. 2012, 13, 2249.

39. Sun, J.; Yue, Y. D.; Tang, F.; Guo, X. F. J. Planar Chromatogr. 2010, $23,40$.

40. Lv, Z. L.; Dong, J.; Zhang, B. L. Bioresources 2012, 7, 1405.

41. Liu, M. H.; Ko, C. H.; Ma, N.; Tan, P. W.; Fu, W. M.; He, J. Y. J. Funct. Foods 2016, 22, 533.

42. Wang, J.; Yue, Y. D.; Tang, F.; Sun, J. Molecules 2012, 17, 12297.

43. Wei, Q.; Yue, Y. D.; Tang, F.; Sun, J.; Wang, S. Y.; Yu, J. Nat. Prod. Res. Dev. 2014, 1, 38.

44. Van Hoyweghen, L.; Karalic, I.; Van Calenbergh, S.; Deforce, D.; Heyerick, A. J. Nat. Prod. 2010, 73, 1573.

45. Sun, J.; Yue, Y. D.; Tang, F.; Guo, X. F. J. Chil. Chem. Soc. 2010, 55, 363.

Open Access. This is an open-access article distributed under the terms of the Creative Commons Attribution-NonCommercial 4.0 International License (https:// creativecommons.org/licenses/by-nc/4.0/), which permits unrestricted use, distribution, and reproduction in any medium for non-commercial purposes, provided the original author and source are credited, a link to the CC License is provided, and changes - if any - are indicated. 